\title{
Dimensions of family disruption: Coincidence, interactions, and impacts on children's educational attainment
}

\author{
Juli Simon Thomas Harvard University, USA \\ jsimonthomas@mitre.org
}

(Received November $2016 \quad$ Revised January 2018)

http://dx.doi.org/10.14301/Ilcs.v9i2.436

\begin{abstract}
Household composition, economic resources, and residence are not necessarily stable across childhood. Changes in parental relationship status, parental employment, and residence have been shown to affect children's educational attainment. Less studied is the fact that these events can occur in combination: families could experience more than one of these disruptive events within the same time period (e.g. year); from a life course perspective, families could experience multiple events throughout their lives. Using linear regression models to analyse data from the Panel Study of Income Dynamics, a longitudinal study of U.S. individuals, I confirmed that the children of parents who experienced employment loss or gain, or partner loss or gain demonstrated lowered odds of high school completion, college attendance, and college completion. Residential moves increased the odds of high school completion but decreased chances of college completion. I then found that experiencing two disruptive events within a given two-year period led to an increased negative effect compared to experiencing only one event. These findings robustly applied to different comparison group specifications. Finally, I showed that, generally, increasing the number of disruptive events decreased the probability of attaining the educational outcomes considered.
\end{abstract}

\section{Keywords}

Education; family; life course 


\section{Introduction}

Household composition, economic resources, and residence are not necessarily stable across childhood. Changes in parental relationship status, parental employment, and residence have been shown to affect children's educational attainment. Research usually focuses on one life event, such as divorce, a move, or job loss; such studies give insights into the dynamics and consequences of isolated events. Less studied is the fact that these events may occur in combination. The possibility that events cascade, triggering subsequent disruption and reinforcement-in which one disruption magnifies the impacts of other disruptions experienced simultaneously-means that family disruption might have second-order effects on children's educational attainment. There are indeed events that prompt another event, such as a residential move following a divorce (Clark, 2016; South, Crowder, \& Trent, 1998; Weitzman, 1985), or a job loss leading to a divorce (Charles \& Stephens, 2004; Sayer, Allison, England, \& Kangas, 2011). Events could also occur independently but successively within a given two-year timeframe. Disruptive events could interact to form their own dynamic relationship, in which consequences can be attributed to both events or to a complex interplay of events. As Werner and Smith (1992) point out, "the intercorrelations among a number of concurrent stressors in children's lives and possible common antecedents....are often overlooked in...investigations" (154). The aim of this study was to examine these potential event combinations and their prevalence and assess the second-order effects of combined disruptions above and beyond the better-known firstorder impacts of singular events on children's educational outcomes.

Treating each event separately allows research to focus and delve more deeply into each event's impact on the life course. However, a focus on singular events only gives a decontextualised view of each event. A central tenet of life course theory holds that any event occurs in the life course as part of a series of events (Elder, 1999; Mayer, 2009) or an age-graded pattern (Elder, Johnson, \& Crosnoe, 2004). Some events are considered part of normative life course stages (e.g. completion of schooling, first marriage, retirement (Riley \& Riley, 1994; Uhlenberg \& Mueller,
2004)), but people additionally experience events that disrupt the life course in some way and create an off-time and sometimes unexpected transition (e.g. from employed to unemployed (McLeod \& Almazan, 2004)) or turning point (Wheaton \& Gotlib, 1997). Disruptive events can be, and often are, coupled together. If each event matters, the interaction between or combination of events may matter as well: for example, if a divorce seemingly prompts a negative outcome, it could be that a residential move coinciding with the divorce is partially causative in the fallout. Prior research has not yet addressed this question, leaving a potentially large gap in the literature. Some studies have focused on cooccurrence of events that mark the transition to adulthood, the increasing individualisation of the life course, and how event sequence patterns are affected (Buchmann, 1989; Rindfuss, Swicegood, \& Rosenfeld, 1987; Shanahan 2000). However, these conversations about event combinations focus on normative life course events, or role transitions, as shaping a transition into adulthood (or parenthood), not disruptive events that occur within the adult life course. Some literature in social psychology has investigated how clusters of adversities or traumas differ from single events (Kessler, Gillis-Light, Magee, Kendler, \& Eaves, 1997) and how families might bear this burden as a collective entity (Malia, 2006), though this work focuses primarily on mental health outcomes.

This paper focuses on the probability of high school graduation, college attendance, and college completion for the children of parents who experienced disruptive life events. Of course, many educational outcomes occurring between birth and high school graduation could be measured, such as grades, disciplinary actions or grade retention. I chose to focus on high school graduation, college attendance and college completion as educational markers that impact a child's likelihood of experiencing the same disruptive events in their subsequent life trajectories. High school diplomas are necessary for the vast majority of jobs in the U.S., and college degrees lead to significantly increased incomes, especially over a lifetime of employment (Borgen \& Rumbaut, 2011). These crucial milestones also provide a protective effect against job loss (Brand \& Simon Thomas, 2014; Kogan, Unt \& Saar, 
2007 and marital instability (Furstenberg, 1995). Indeed, I show the impact of parents' educational milestones on their own likelihood to experience disruptive events as well. If disruptive events in the parents' lives create situations in which children are more likely to experience the same events owing to the children missing educational milestones, this provides strong evidence for an intergenerational pathway of disadvantage.

\section{Background}

Previous research shows that disruptive events indeed matter for children's educational attainment; usually effects are negative, though income can be protective (Mortimer, Zhang, Husseman \& Wu, 2014; Ryan, Claessens \& Markowitz, 2013). Parental divorce or dissolution of a cohabiting relationship (Amato, 2000; Amato \& Keith, 1991; Brown, 2010; Cooper, Osborne, Beck, \& McLanahan, 2011; Fomby \& Sennott, 2013; Garfinkel \& McLanahan, 1986; Mitchell et al., 2015; Rhodes \& Hoey, 1994; Seltzer, 1994; Sweeney, 2011; Thomas \& Högnäs, 2015; Werner \& Smith, 1992), remarriage or the start of a cohabiting relationship (Brown, 2010; Fomby \& Sennott, 2013; Jaffee, Moffitt, Caspi \& Taylor, 2003; Mitchell et al. 2015; Sweeney, 2011), residential moves (Burdick-Will et al., 2011; DeWit, 1998; Ingersoll, Scamman \& Eckerling, 1989; Jelleyman \& Spencer, 2008 Pettit \& McLanahan, 2003; South, Haynie \& Bose, 2007) and job loss (Brand \& Simon Thomas, 2014; Parsons, Schoon \& Vignoles, 2014) can negatively affect children educationally, socially, and emotionally. Combinations of events are probable: divorce or ending cohabitation has been shown to lead to residential moves (Swartz, Hartmann \& Mortimer, 2011; Weitzman, 1985), similar to remarriage or the start of a new cohabiting relationship.

There are various pathways by which children's educational attainment can be affected by disruptive events. One is purely economic: divorce and job loss specifically negatively affect family income (Davis \& von Wachter, 2011; Tach \& Eads, 2014; Tach \& Eads, 2015), and fluctuations in income have been shown to have negative consequences for children's education (Gennetian, Wolf, Hill, \& Morris, 2015). Single mothers' job losses have been shown to lead to lower rates of high school and college completion for their children, and the authors (Brand \& Simon Thomas, 2014) suggest that relative deprivation plays a role in these effects. Amato and Booth (1991) refer to this as an "economic deprivation" model. This economic argument would imply that marriage (or remarriage) and job gain would lead to positive results, given the likely increase in financial resources (Hao, 1996). Importantly, family income could also act as a buffer to the consequences of marital instability (Ryan, Claessens \& Markowitz, 2013.

An alternative argument is that parental life disruptions could harm children by affecting their daily lives, such as partner status changes that lead to changes in childcare, which is disruptive for young children (Crosnoe, Chambers Prickett, Smith, \& Cavanagh, 2014). ${ }^{1}$ Corak (2004) argues directly that income is not the driving factor of disruptions' effects: "The decisive kinds of parental investments are not the monetary kind. The inheritance of education, occupation, and income is influenced in the first instance by the impact parents have on a child's cognitive performance" (Corak, 2004:33). By Corak's logic, changes in income, or even in overall socioeconomic status (e.g. occupational change and income change), should not change children's educational choices because these disruptive events do not change parents' cognitive influences. The parents remain the same (though divorce will alter exposure and stepfamily formation adds new people to the household), regardless of different employment situations, different relationships and/or living in a different home. However, I believe that parental disruptions necessarily impact children in that they can add stress and change income.

Disruptive events, such as partner exits and entrances (Amato \& Booth, 1991; Amato \& Keith, 1991; Mitchell et al., 2015) or job losses (Paul \& Moser, 2009; Young, 2012), are also stressful for parents and their children, and this stress can affect behaviour and mental health. Pearlin, Menaghan, Lieberman, and Mullan (1981) argue that "unscheduled or undesired life events" (343), or "eventful experiences" (338) lead to role strain, or problems in the roles one is expected to play socially and personally, which in turn affects self-esteem and feelings of mastery. Pearlin more recently suggests that agency is diminished by life course disruptions, with potential negative effects (Pearlin, 2010). Both 
mastery and agency mean that individuals feel in control over possible transition points in their lives and their consequences, and disruptive events impinge upon these feelings of control. When parents experience stress, it is likely to affect their children: "the actions, fortunes, and misfortunes of one person are likely to affect those with whom the person has close social relationships" (Pearlin 2010: 212). The family stress model underscores the idea that stressful life events take time to churn through each family member, potentially creating a complex interaction of emotions and reactions, or "upset in the steady state of the family" (Malia, 2006: 143). When children's mental health and behaviour (particularly classroom behaviour) suffers, this can affect their educational attainment, among other factors.

More generally, recent research likewise shows that instability in families is detrimental to children's educational performance (Cavanagh \& Fomby, 2011), college completion (Fomby, 2013), behaviour (Cavanagh \& Huston, 2006; Fomby \& Sennott, 2013; Mitchell et al., 2015; Ryan, Claessens \& Markowitz, 2014), and other outcomes related to the transition to adulthood, such as union formation, childbearing and entry into the labour force (Fomby \& Bosick, 2013). The experience of multiple disruptive events within close time proximity is akin to instability. Research on families suggests that instability and change might be more to blame for negative effects on children rather than the family statuses themselves (Osborne \& McLanahan, 2007; Sweeney, 2010; Wu \& Martinson, 1993), over and above the negative effects of income changes (Wu, 1996). Indeed, the negative effects of family instability affect children across all levels of income (Ryan, Claessens \& Markowitz, 2014).

However, a recent report by the Urban Institute (2014) points out that though instability is negative, there could be changes that create a form of instability but ultimately lead to better outcomes, such as a residential move when a parent gets a better job. Importantly, they also state that "the frequency or repetition of the experience of instability for children is an important consideration; a single experience of instability seems likely to have a different effect on children than repeated incidences of instability" (2). In an overview of related research, they refer to such repeated instances as a "cascade of instability," in which one event prompts another event. This is akin to the idea of a chain reaction within a life trajectory (Wheaton \& Gotlib, 1997) or a cluster of adversities (Kessler et al., 1997).

There is already ample support for the hypothesis that parental disruptions negatively affect children's educational attainment. But whether and how combinations of events, as compared to single events, affect children's educational attainment remains unclear, though we can build the inference from existing research on single events that the effects of event combinations should be negative. If research on independent events is used as a guide, we could think of the consequences of multiple events as being additive. That is, a child whose parents divorce and then move experiences the negative consequences of the divorce plus the negative consequences of the residential change. I refer to this possible combination of event effects as the additive hypothesis.

Another possibility is that the effects of disruptions are not independent but rather interact to form increasingly exacerbated circumstances. Time proximity could lead to effects worsening beyond a simply additive process: in other words, there is the effect of each event as well as an additional increase in effects that can be attributed to event or effect interaction. Conversely, event effects could overlap, resulting in a total effect that is less than a simple addition of effects from separate events. I call this potentially multiplicative combination of event effects the amplification hypothesis, in which the multiplicative factor could be greater or less than 1.

To test these two hypotheses, I first asked: How often are events found in combination with one another? I also evaluated which combinations were most common (i.e. which events were often experienced in the same family within a two-year time frame). These questions focused on the parents' experiences with events to see how normative these event combination experiences might be. To focus on the effects specifically, I asked: Do certain event combinations lead to lower educational outcomes for children than others? Relatedly, do any of these combinations of events lead to positive educational outcomes for children? 
A final hypothesis I considered stems from the idea of instability becoming normative in some households: it is possible that there is a point at which the effects of disruptive events are diminished by their combination and repetition. This means the outcomes are less about the exact events that occur and more about the quantity of them; the life course becomes defined in part by these disruptions. This is a more qualitative view of the simultaneity of events, which I refer to as the cumulation hypothesis. To test this final hypothesis, I asked: Is a higher count of events worse (in terms of children's educational outcomes) than a lower count? I was specifically interested in a possible non-linear trend as event count increased.

\section{Data}

I used data from the Panel Study of Income Dynamics (PSID) for this analysis. The PSID began in 1968 as an annual survey, and since 1997 it has continued to add data every two years. The study began with a sample of 18,000 individuals in 5,000 families (these participants are said to have the "PSID gene" or be "gened participants"), and the study is still growing as family members are added, creating a sample by 2011 of over 80,000 individuals. The PSID contains detailed information about job changes, partnership status, and residential location. It also contains, at a detailed level, a rich set of covariates for this analysis.

Initially, my sample included 22,104 children matched with parents. I removed respondents who were not at least 19 years old by $2011(n=6,461)$, as those respondents had not reached the cut-off age of high school completion. (Changing this cut-off to 21 or 25 did not change results, so I used the dataset with a larger number of respondents, which used the cut-off of being at least 19 years old in 2011.) I only analysed events that occurred when the child was between 1 and 17 years of age. I removed respondents for whom data on any disruptive event for mothers or fathers, for all years, was missing $(n=4,261)$, as leaving those cases in the dataset would have required substantial imputation across many years on my main predictive variables. Finally, across mothers and fathers with employment, relationship, and residential move data available, I retained 11,382 children in my sample.
I used a subset of the trigger events that DiPrete and McManus (2000) used. Though these authors looked at different outcomes, I believe these events captured instability and change in socioeconomic status for parents and hence their dependent children, which was appropriate for this analysis. The employment events were: (1) employment to no employment, (2) no employment to employment, and the relationship events were: (1) add partner, and (2) lose partner. It is important to note that the PSID classifies respondents as "married" if they have been cohabiting for more than a year; therefore, I used this variable indicating being "married" in my analyses. Thus, I focused on unions that dissolved or were entered into whether they were married or cohabiting, following the PSID definition of relationship length. I did not consider girlfriends and boyfriends in the household, as I believe that marriage or long-term cohabitation dissolution provides a greater shock to the household, and it was such large shocks in which I was interested. Though cohabitation and marriage have become increasingly similar in behaviour and effects on children (Goldberg \& Carlson, 2014; Tach \& Eads, 2014; Tach \& Eads, 2015), I acknowledge that this is a generalisation and there could be differences for children whose parents have cohabiting versus married relationships. I also included a third event category: moving to a new residence, which made use of a question in the PSID in which respondents were asked if they had moved in the previous year. This gave me a total of five events to analyse, ${ }^{2}$ which I only coded as occurring if there was a child in the household between the ages of 1 and 17 years. This list was by no means exhaustive; many more events could have been considered disruptive to families. This analysis was meant to provide a starting point with some of the most important trigger events for families. This line of research can be extended to other events, such as health-related changes (e.g. severe illnesses or death in families).

I measured educational outcomes for children following Brand and Simon Thomas (2014) as high school completion by age 19 , college attendance by age 21 , and college completion by age 25 . Notably, results for each educational outcome could be quite different, because college usually requires a financial investment and attendance is not mandatory at any 
age; persistence to college completion might also vary from attendance since it requires attending for multiple years and completing a set of requirements.

I primarily focused on event occurrence for either parent. However, following arguments that both parents' class origins matter for children and must be taken into account when analysing intergenerational mobility (Beller, 2009), I also analysed mothers' and fathers' events separately for comparison. The PSID's unique design uses a "head of household" designation, which in the early years of the survey meant the male adult respondent in the household. However, in households where the adult present was female, for example in the aftermath of a divorce, the female adult respondent became the head of household. Conversely, a marriage meant that head of household shifted to the male respondent. Due to these potential shifts, I placed heavier emphasis on the outcomes for parents generally to draw conclusions.

Finally, as is the case in most longitudinal surveys, attrition must be addressed. In the earlier half of the PSID, 2-3\% of the sample was lost per year due to attrition (Falaris \& Peters, 1998). Attrition could have occurred due to mortality. Additionally, if non-sample members left the household, they were no longer followed beyond that point in time; this means that relationship dissolution could have led to survey follow-up loss. More generally, an ended relationship could mean that survey participants were lost even if they were gened participants, because they moved and could not be located or they refused continued participation. Between 1968 and 1996, 5-13\% of PSID fathers became non-resident, depending on the decade (Gupta, Smock \& Manning, 2004). If attrition is selective - which evidence suggests it is, based on education, income, and health (Fitzgerald, 2011; Hofferth, 2006; Schoeni \& Wiemers, 2015) - then this could lead to systematic variation and bias results. Because I removed respondents for whom data on disruptive events was missing, those respondents who did not have this event data for fathers were removed from the sample. Thus, because some of the less stable families could ultimately be missing from the sample, and these might be the families showing stronger effects of disruption, it is possible that results were slightly underestimated. However,
Fitzgerald (2011) finds no evidence of the impact of attrition on outcomes in intergenerational models.

\section{Methods}

To code events, I created dichotomous variables. Prior to 1996, every year was included; post-1996, data were available for every other year. For the "employment to no employment" variable, I looked at employment in two adjacent years to see if there was a change from employed to not employed; if this was the case, the "employment to no employment" variable was coded as 1 rather than 0 . Those who did not experience the event for any reason (including possibly being already not employed) are coded as zero. This comparison group is intentionally left to be general and inclusive, in order to allow for aggregation over the years included, to emphasise the "event-ness" rather than the specific nature of the particular change, and to include all respondents in the possible selection group when events are combined. (If one year's variable relevant to the event variable was missing, the event variable itself was coded as missing for that individual.) The other events were coded in a similar way. Thus, the events variables were coded as ever having experienced the event, with the boundaries being the time of the survey (1968-2011) and the child's age (1-17 years old).

I remained agnostic as to the underlying reasons for the responses (e.g. employed) and thus was looking at people whose employment or marital statuses changed for any reason, so long as I could view a change across years. (I did not capture those people who switched jobs in the middle of a year, for example - i.e. those people would lose and gain a job within the same two-year period considered.) Again, given the switch to biannual data collection post1997, I considered events and event combinations across every two years rather than annually; this means that pre-1997 data was condensed to twoyear intervals. In doing this, I found that event occurrences were balanced across two-year time spans, indicating that data collection efforts post1997 reflected coverage of two years rather than one year.

To code events combinations, I created a variable for two events occurring in the same two years. I did not analyse the reasons or ordering for combinations 
but rather looked to see if events occurred within the same two years. Occurring within such close time proximity meant children were exposed to more than one event within a short time frame, and the impact of that combination was of interest. It was possible that one event prompted another event, although their close proximity in time could also be coincidental; I could not determine which event occurred first when they occurred in the same survey year (where survey years are every two years) nor the exact reasons for their time proximity. Regardless of reason or order of coincidence, the fact remains that parents, and hence their children, were subject to the effects of both events within the same two-year time period. I defined this as a set of combined disruptive events.

I limited my sample to children who were at least 19 years old by the latest survey date (in 2011). I also looked only at parental events that occurred when their surveyed child was between 1 and 17 years old; events that occurred prior to that child's birth and once the child was at least 18 years old were not included in my analysis. There is a chance that some children were also parents with surveyed children in the sample; I did not exclude these cases. (These cases could be examined as a separate study in future work, though sample sizes would likely be quite limited, even using data from a study as large as the PSID.) There was some censoring of children who were born prior to 1968; they were included in the analysis (unless they turned 18 prior to 1968), although I only observed events that occurred in the years of their childhood that were included in the survey. Approximately $32 \%$ of children included in the survey were born prior to 1968, and thus, their childhood years are somewhat truncated. This means that my sample of children was slightly skewed toward older child ages as compared to a sample in which all childhood years would be included for all children; ages ranged from 19 to 61 years old in 2011 for the final sample. For children born prior to 1968, variables coded as a status at birth (e.g. mother's marital status) used this status in 1969 instead. (Variables in 1969 had fewer missing cases than 1968. For the non-missing cases, there was little difference between values in 1968 and 1969, so I opted to use the variables with fewer missing cases.)
I used linear regression models to see which covariates significantly predicted incidences of disruptive events in mothers' and fathers' lives. (These models were also run as logistic regression models and as Poisson models, and I found no differences in effects in terms of which covariates significantly affect the events examined. Thus, I used linear regression models for simplicity.) Linear regression models took the form of:

$$
y=\beta_{0}+\beta_{1} * x_{1}+\beta_{2} * x_{2}+\ldots+\beta_{n} * x_{n}
$$

where $y$ was the probability of the disruptive event, $x_{n}$ was the set of covariates being examined in this model, $\beta_{0}$ was the constant, and the other $\beta_{n}$ 's signified the set of coefficients for each variable. I examined the $\beta_{n}$ coefficients to see which covariates significantly predicted events.

I used logistic regression models to estimate effects of parental events on child educational outcomes. Logistic models took the form of:

$$
\log \left(\frac{p}{1-p}\right)=\beta_{0}+\beta_{1} * x_{1}+\beta_{2} * x_{2}+\ldots+\beta_{n} * x_{n}
$$

where $p$ was the probability of reaching an educational milestone (completing high school by age 19 , attending college by age 21 , or completing college by age 25), $x_{n}$ was the set of covariates being examined in this model, $\beta_{0}$ was the constant, and the other $\beta_{n}$ 's signified the set of coefficients for each variable. Each educational outcome was a separate model; each event was considered in a separate model as well. I analysed mothers' and fathers' events in separate models to be able to examine potential differences in parental influence. Covariates for all models included the child's sex and race, whether the child was born in the U.S. South, mother's and father's high school completion, mother's and father's college completion; I also included covariates signifying if the mother or father was married when their child was born, and covariates signifying if the mother or father worked in manufacturing when their child was born. Earlier models included a control variable for parental age at child's birth; this variable was not significant and did 
not change the results at all, and was therefore removed to maintain simpler models.

Descriptive statistics are shown in table 1 for events for parents. Children did appear to have lower educational attainment, on average, when one of their parents experienced an event. Educational attainment for both mothers and fathers was also lower among those who experienced events, as was the chance of being married when the child was born. Working in manufacturing did not show a pattern, though there were, on average, differences within each event. Descriptive statistics for mothers and fathers, shown in summary form in table 2 and in full in appendices $A$ and $B$, showed similar patterns except for an important distinction: high school completion for children was largely the same whether or not mothers experienced disruptive employment events, whereas when fathers experienced employment events, the chance of any of the children's educational outcomes was lower, as might be expected. Whereas the presence of partnership change events meant a lower chance of any educational outcomes, moving actually led to slightly higher means of children's high school completion and college attendance, though lower means of college completion; these patterns held true for both mothers' and fathers' events. Education also appeared protective for fathers only: higher educational attainment was protective against experiencing events, whereas higher educational attainment did not afford mothers the same protection. Because of these interesting differences, I looked at mothers and fathers separately again in other analyses to see what this may have meant for children.

Finally, in other work I showed that both the age of children and the time placement of the event(s) in calendar years could affect outcomes (Simon Thomas, n.d.) In this manuscript, however, I was interested in the overall effects; breaking down the analysis by these two factors is addressed in additional work.

\section{Results}

\section{Analysis of events}

I first focused on exploratory analyses to overcome the lack of coverage in the literature on how these life events might combine. These initial analyses involved determining how often events occurred and then how often events occurred in close temporal proximity. To start, I looked at the frequency of event occurrence in respondents' lives. Disruptive event occurrence, generally, was not rare. As shown in table 3 , the frequency of respondents experiencing single events for parents was $34 \%$ and $33 \%$ for employment loss and gain, respectively, and $28 \%$ and $24 \%$ for partner loss and gain, respectively. Over $70 \%$ of respondents experienced a residential move of some kind during the time period considered. Single events for mothers and fathers alone were only slightly less in number. With a sample size of 11,382, this gave a more than adequate number of event occurrences for analysis; it also made it likely that respondents would experience more than one event during their children's childhood years, particularly in combination with residential moves, given their frequency.

Indeed, between $15 \%$ and $21 \%$ of respondents experienced employment and marital events combined with residential moves in the same twoyear period at some point during their childhood. For mothers and fathers, between $9 \%$ and $19 \%$ experienced these event combinations. Employment events combined with marital events in the same time period were less frequent, at $4-5 \%$ for parents and $2-3 \%$ for mothers and fathers. This shows that event combinations were not uncommon; many families experienced two events in close temporal proximity.

Next, I investigated demographic characteristics that might impact the likelihood of experiencing a disruptive event. Initial linear regression models revealed the covariates that had significant bearing on disruptive event occurrence; results for parents and children are shown in table 4 . Non-white parents were more likely to experience all events. Children born earlier in the sample were less likely to have 


\begin{tabular}{|c|c|c|c|c|c|c|c|c|c|c|c|}
\hline & \multirow{2}{*}{ All children } & \multicolumn{2}{|c|}{ Employment loss } & \multicolumn{2}{|c|}{ Employment gain } & \multicolumn{2}{|c|}{ Partner loss } & \multicolumn{2}{|c|}{ Partner gain } & \multicolumn{2}{|c|}{ Moved } \\
\hline & & Yes & No & Yes & No & Yes & No & Yes & No & Yes & No \\
\hline & $\begin{array}{l}\text { Mean } \\
\text { (SD) }\end{array}$ & $\begin{array}{c}\text { Mean } \\
\text { (SD) }\end{array}$ & $\begin{array}{c}\text { Mean } \\
(S D)\end{array}$ & $\begin{array}{c}\text { Mean } \\
\text { (SD) }\end{array}$ & $\begin{array}{l}\text { Mean } \\
(S D)\end{array}$ & $\begin{array}{l}\text { Mean } \\
(S D)\end{array}$ & $\begin{array}{c}\text { Mean } \\
\text { (SD) }\end{array}$ & $\begin{array}{l}\text { Mean } \\
(S D)\end{array}$ & $\begin{array}{c}\text { Mean } \\
(S D)\end{array}$ & $\begin{array}{c}\text { Mean } \\
(S D)\end{array}$ & $\begin{array}{c}\text { Mean } \\
(S D)\end{array}$ \\
\hline \multicolumn{12}{|l|}{ Child variables } \\
\hline $\begin{array}{l}\text { High school completion } \\
\text { by age } 19\end{array}$ & $\begin{array}{c}0.484 \\
(0.500)\end{array}$ & $\begin{array}{c}0.477 \\
(0.500)\end{array}$ & $\begin{array}{c}0.487 \\
(0.500)\end{array}$ & $\begin{array}{c}0.476 \\
(0.499)\end{array}$ & $\begin{array}{c}0.488 \\
(0.500)\end{array}$ & $\begin{array}{c}0.460 \\
(0.498)\end{array}$ & $\begin{array}{c}0.493 \\
(0.500)\end{array}$ & $\begin{array}{c}0.466 \\
(0.499)\end{array}$ & $\begin{array}{c}0.489 \\
(0.500)\end{array}$ & $\begin{array}{c}0.507 \\
(0.500)\end{array}$ & $\begin{array}{c}0.429 \\
(0.495)\end{array}$ \\
\hline $\begin{array}{l}\text { College attendance } \\
\text { by age } 21\end{array}$ & $\begin{array}{c}0.259 \\
(0.438)\end{array}$ & $\begin{array}{c}0.239 \\
(0.427)\end{array}$ & $\begin{array}{c}0.269 \\
(0.443)\end{array}$ & $\begin{array}{c}0.240 \\
(0.427)\end{array}$ & $\begin{array}{c}0.268 \\
(0.443)\end{array}$ & $\begin{array}{c}0.214 \\
(0.410)\end{array}$ & $\begin{array}{c}0.277 \\
(0.447)\end{array}$ & $\begin{array}{c}0.215 \\
(0.411)\end{array}$ & $\begin{array}{c}0.272 \\
(0.445)\end{array}$ & $\begin{array}{c}0.270 \\
(0.444)\end{array}$ & $\begin{array}{c}0.233 \\
(0.423)\end{array}$ \\
\hline $\begin{array}{l}\text { College completion } \\
\text { by age } 25\end{array}$ & $\begin{array}{l}0.130 \\
(0.336)\end{array}$ & $\begin{array}{l}0.095 \\
(0.293)\end{array}$ & $\begin{array}{c}0.148 \\
(0.355)\end{array}$ & $\begin{array}{c}0.098 \\
(0.298)\end{array}$ & $\begin{array}{c}0.146 \\
(0.353)\end{array}$ & $\begin{array}{c}0.079 \\
(0.270)\end{array}$ & $\begin{array}{c}0.150 \\
(0.357)\end{array}$ & $\begin{array}{l}0.078 \\
(0.268)\end{array}$ & $\begin{array}{c}0.146 \\
(0.353)\end{array}$ & $\begin{array}{c}0.121 \\
(0.326)\end{array}$ & $\begin{array}{c}0.152 \\
(0.359)\end{array}$ \\
\hline Male $(0 / 1)$ & $\begin{array}{c}0.512 \\
(0.500)\end{array}$ & $\begin{array}{c}0.516 \\
(0.500)\end{array}$ & $\begin{array}{c}0.510 \\
(0.500)\end{array}$ & $\begin{array}{c}0.515 \\
(0.500)\end{array}$ & $\begin{array}{c}0.511 \\
(0.500)\end{array}$ & $\begin{array}{c}0.510 \\
(0.500)\end{array}$ & $\begin{array}{c}0.513 \\
(0.500)\end{array}$ & $\begin{array}{c}0.517 \\
(0.500)\end{array}$ & $\begin{array}{c}0.510 \\
(0.500)\end{array}$ & $\begin{array}{c}0.514 \\
(0.500)\end{array}$ & $\begin{array}{c}0.506 \\
(0.500)\end{array}$ \\
\hline Black (0/1) & $\begin{array}{c}0.331 \\
(0.471)\end{array}$ & $\begin{array}{c}0.415 \\
(0.493)\end{array}$ & $\begin{array}{c}0.287 \\
(0.453)\end{array}$ & $\begin{array}{c}0.421 \\
(0.494)\end{array}$ & $\begin{array}{c}0.287 \\
(0.452)\end{array}$ & $\begin{array}{c}0.440 \\
(0.496)\end{array}$ & $\begin{array}{c}0.287 \\
(0.453)\end{array}$ & $\begin{array}{c}0.458 \\
(0.498)\end{array}$ & $\begin{array}{c}0.291 \\
(0.454)\end{array}$ & $\begin{array}{c}0.359 \\
(0.480)\end{array}$ & $\begin{array}{c}0.262 \\
(0.440)\end{array}$ \\
\hline Other race $(0 / 1)$ & $\begin{array}{c}0.083 \\
(0.276)\end{array}$ & $\begin{array}{c}0.086 \\
(0.281)\end{array}$ & $\begin{array}{c}0.081 \\
(0.273)\end{array}$ & $\begin{array}{c}0.092 \\
(0.289)\end{array}$ & $\begin{array}{c}0.079 \\
(0.269)\end{array}$ & $\begin{array}{c}0.095 \\
(0.294)\end{array}$ & $\begin{array}{c}0.078 \\
(0.268)\end{array}$ & $\begin{array}{c}0.098 \\
(0.297)\end{array}$ & $\begin{array}{c}0.078 \\
(0.269)\end{array}$ & $\begin{array}{c}0.086 \\
(0.281)\end{array}$ & $\begin{array}{c}0.075 \\
(0.263)\end{array}$ \\
\hline Age in 2011 & $\begin{array}{c}36.090 \\
(11.755)\end{array}$ & $\begin{array}{l}31.779 \\
(8.585)\end{array}$ & $\begin{array}{c}38.316 \\
(12.527)\end{array}$ & $\begin{array}{l}31.236 \\
(8.358)\end{array}$ & $\begin{array}{c}38.461 \\
(12.427)\end{array}$ & $\begin{array}{l}32.173 \\
(9.594)\end{array}$ & $\begin{array}{c}37.629 \\
(12.163)\end{array}$ & $\begin{array}{l}31.645 \\
(8.856)\end{array}$ & $\begin{array}{c}37.462 \\
(12.192)\end{array}$ & $\begin{array}{c}33.898 \\
(10.178)\end{array}$ & $\begin{array}{c}41.420 \\
(13.498)\end{array}$ \\
\hline Born in South & $\begin{array}{c}0.389 \\
(0.488)\end{array}$ & $\begin{array}{c}0.418 \\
(0.493)\end{array}$ & $\begin{array}{c}0.375 \\
(0.484)\end{array}$ & $\begin{array}{c}0.425 \\
(0.494)\end{array}$ & $\begin{array}{c}0.372 \\
(0.483)\end{array}$ & $\begin{array}{c}0.464 \\
(0.499)\end{array}$ & $\begin{array}{c}0.360 \\
(0.480)\end{array}$ & $\begin{array}{c}0.461 \\
(0.499)\end{array}$ & $\begin{array}{c}0.367 \\
(0.482)\end{array}$ & $\begin{array}{c}0.421 \\
(0.494)\end{array}$ & $\begin{array}{c}0.312 \\
(0.463)\end{array}$ \\
\hline \multicolumn{12}{|l|}{ Mother variables } \\
\hline High school completion & $\begin{array}{c}0.170 \\
(0.375)\end{array}$ & $\begin{array}{c}0.187 \\
(0.390)\end{array}$ & $\begin{array}{c}0.161 \\
(0.367)\end{array}$ & $\begin{array}{c}0.186 \\
(0.389)\end{array}$ & $\begin{array}{c}0.162 \\
(0.368)\end{array}$ & $\begin{array}{c}0.166 \\
(0.363)\end{array}$ & $\begin{array}{c}0.175 \\
(0.380)\end{array}$ & $\begin{array}{c}0.178 \\
(0.383)\end{array}$ & $\begin{array}{c}0.167 \\
(0.373)\end{array}$ & $\begin{array}{c}0.177 \\
(0.381)\end{array}$ & $\begin{array}{c}0.153 \\
(0.360)\end{array}$ \\
\hline Some college & $\begin{array}{c}0.104 \\
(0.305)\end{array}$ & $\begin{array}{c}0.132 \\
(0.338)\end{array}$ & $\begin{array}{c}0.089 \\
(0.285)\end{array}$ & $\begin{array}{c}0.140 \\
(0.348)\end{array}$ & $\begin{array}{c}0.085 \\
(0.280)\end{array}$ & $\begin{array}{c}0.128 \\
(0.334)\end{array}$ & $\begin{array}{c}0.094 \\
(0.292)\end{array}$ & $\begin{array}{c}0.135 \\
(0.342)\end{array}$ & $\begin{array}{c}0.094 \\
(0.291)\end{array}$ & $\begin{array}{c}0.120 \\
(0.325)\end{array}$ & $\begin{array}{c}0.064 \\
(0.244)\end{array}$ \\
\hline College completion & $\begin{array}{c}0.092 \\
(0.290)\end{array}$ & $\begin{array}{c}0.079 \\
(0.270)\end{array}$ & $\begin{array}{c}0.099 \\
(0.299)\end{array}$ & $\begin{array}{c}0.086 \\
(0.280)\end{array}$ & $\begin{array}{c}0.095 \\
(0.294)\end{array}$ & $\begin{array}{c}0.063 \\
(0.242)\end{array}$ & $\begin{array}{c}0.104 \\
(0.305)\end{array}$ & $\begin{array}{c}0.066 \\
(0.249)\end{array}$ & $\begin{array}{c}0.100 \\
(0.300)\end{array}$ & $\begin{array}{c}0.103 \\
(0.303)\end{array}$ & $\begin{array}{c}0.067 \\
(0.251)\end{array}$ \\
\hline Married@ child born & $\begin{array}{c}0.918 \\
(0.274)\end{array}$ & $\begin{array}{c}0.869 \\
(0.338)\end{array}$ & $\begin{array}{c}0.945 \\
(0.228)\end{array}$ & $\begin{array}{c}0.864 \\
(0.343)\end{array}$ & $\begin{array}{c}0.946 \\
(0.226)\end{array}$ & $\begin{array}{c}0.837 \\
(0.369)\end{array}$ & $\begin{array}{c}0.953 \\
(0.213)\end{array}$ & $\begin{array}{c}0.716 \\
(0.451)\end{array}$ & $\begin{array}{c}0.987 \\
(0.112)\end{array}$ & $\begin{array}{c}0.894 \\
(0.308)\end{array}$ & $\begin{array}{c}0.986 \\
(0.118)\end{array}$ \\
\hline Work in manufacturing & 0.043 & 0.041 & 0.044 & 0.041 & 0.044 & 0.037 & 0.046 & 0.034 & 0.046 & 0.040 & 0.052 \\
\hline
\end{tabular}




\begin{tabular}{|c|c|c|c|c|c|c|c|c|c|c|c|}
\hline @ child born & $(0.203)$ & $(0.200)$ & $(0.205)$ & $(0.199)$ & $(0.205)$ & $(0.188)$ & $(0.209)$ & $(0.181)$ & $(0.209)$ & $(0.195)$ & $(0.222)$ \\
\hline \multicolumn{12}{|l|}{ Table 1: Continued. } \\
\hline \multicolumn{12}{|l|}{ Father variables } \\
\hline \multirow[t]{2}{*}{ High school completion } & 0.125 & 0.154 & 0.111 & 0.156 & 0.111 & 0.113 & 0.130 & 0.139 & 0.121 & 0.135 & 0.102 \\
\hline & $(0.331)$ & $(0.361)$ & $(0.314)$ & $(0.363)$ & $(0.314)$ & (0.317) & $(0.337)$ & $(0.346)$ & $(0.326)$ & $(0.342)$ & $(0.303)$ \\
\hline \multirow[t]{2}{*}{ Some college } & 0.071 & 0.073 & 0.069 & 0.078 & 0.067 & 0.066 & 0.073 & 0.082 & 0.067 & 0.078 & 0.053 \\
\hline & $(0.257)$ & $(0.261)$ & $(0.254)$ & $(0.268)$ & $(0.251)$ & (0.249) & $(0.259)$ & $(0.274)$ & $(0.251)$ & (0.269) & $(0.223)$ \\
\hline \multirow[t]{2}{*}{ College completion } & 0.099 & 0.071 & 0.114 & 0.077 & 0.110 & 0.057 & 0.116 & 0.061 & 0.111 & 0.111 & 0.070 \\
\hline & (0.299) & $(0.257)$ & $(0.318)$ & $(0.266)$ & $(0.314)$ & $(0.232)$ & $(0.320)$ & (0.239) & (0.314) & $(0.314)$ & $(0.256)$ \\
\hline \multirow[t]{2}{*}{ Married @ child born } & 0.919 & 0.870 & 0.946 & 0.866 & 0.947 & 0.841 & 0.953 & 0.719 & 0.988 & 0.896 & 0.987 \\
\hline & $(0.272)$ & $(0.336)$ & (0.225) & $(0.341)$ & $(0.223)$ & $(0.366)$ & $(0.211)$ & (0.449) & (0.109) & $(0.306)$ & (0.114) \\
\hline \multirow{2}{*}{$\begin{array}{l}\text { Work in manufacturing } \\
\text { @ child born }\end{array}$} & 0.157 & 0.126 & 0.173 & 0.119 & 0.176 & 0.126 & 0.169 & 0.099 & 0.175 & 0.131 & 0.220 \\
\hline & $(0.364)$ & $(0.332)$ & $(0.378)$ & $(0.324)$ & $(0.381)$ & $(0.332)$ & $(0.375)$ & (0.299) & $(0.380)$ & (0.337) & $(0.415)$ \\
\hline$N$ & 11,382 & 3,876 & 7,506 & 3,736 & 7,646 & 3,211 & 8,171 & 2,685 & 8,697 & 8,065 & 3,317 \\
\hline
\end{tabular}


Table 2: Summary Descriptive Statistics (events for mothers and fathers, separately)

\begin{tabular}{|c|c|c|c|c|c|c|c|c|c|c|}
\hline & \multicolumn{2}{|c|}{ Employment loss } & \multicolumn{2}{|c|}{ Employment gain } & \multicolumn{2}{|c|}{ Partner loss } & \multicolumn{2}{|c|}{ Partner gain } & \multicolumn{2}{|c|}{ Moved } \\
\hline & Yes & No & Yes & No & Yes & No & Yes & No & Yes & No \\
\hline & $\begin{array}{l}\text { Mean } \\
(S D)\end{array}$ & $\begin{array}{l}\text { Mean } \\
(S D)\end{array}$ & $\begin{array}{l}\text { Mean } \\
(S D)\end{array}$ & $\begin{array}{l}\text { Mean } \\
(S D)\end{array}$ & $\begin{array}{l}\text { Mean } \\
(S D)\end{array}$ & $\begin{array}{c}\text { Mean } \\
(S D)\end{array}$ & $\begin{array}{c}\text { Mean } \\
(S D)\end{array}$ & $\begin{array}{c}\text { Mean } \\
(S D)\end{array}$ & $\begin{array}{c}\text { Mean } \\
(S D)\end{array}$ & $\begin{array}{l}\text { Mean } \\
(S D)\end{array}$ \\
\hline \multicolumn{11}{|l|}{ Mother's events } \\
\hline $\begin{array}{l}\text { High school completion } \\
\text { by age } 19\end{array}$ & $\begin{array}{c}0.489 \\
(0.500)\end{array}$ & $\begin{array}{c}0.483 \\
(0.500)\end{array}$ & $\begin{array}{c}0.488 \\
(0.500)\end{array}$ & $\begin{array}{c}0.483 \\
(0.500)\end{array}$ & $\begin{array}{c}0.460 \\
(0.498)\end{array}$ & $\begin{array}{c}0.493 \\
(0.500)\end{array}$ & $\begin{array}{c}0.458 \\
(0.498)\end{array}$ & $\begin{array}{c}0.490 \\
(0.500)\end{array}$ & $\begin{array}{c}0.507 \\
(0.500)\end{array}$ & $\begin{array}{c}0.432 \\
(0.495)\end{array}$ \\
\hline \multicolumn{11}{|l|}{ Mother variables } \\
\hline High school completion & $\begin{array}{c}0.188 \\
(0.391)\end{array}$ & $\begin{array}{c}0.165 \\
(0.371)\end{array}$ & $\begin{array}{c}0.185 \\
(0.389)\end{array}$ & $\begin{array}{c}0.165 \\
(0.372)\end{array}$ & $\begin{array}{c}0.156 \\
(0.363)\end{array}$ & $\begin{array}{c}0.175 \\
(0.380)\end{array}$ & $\begin{array}{c}0.199 \\
(0.399)\end{array}$ & $\begin{array}{c}0.162 \\
(0.369)\end{array}$ & $\begin{array}{c}0.178 \\
(0.382)\end{array}$ & $\begin{array}{c}0.150 \\
(0.357)\end{array}$ \\
\hline Some college & $\begin{array}{l}0.138 \\
(0.345)\end{array}$ & $\begin{array}{l}0.094 \\
(0.292)\end{array}$ & $\begin{array}{l}0.154 \\
(0.361)\end{array}$ & $\begin{array}{l}0.090 \\
(0.287)\end{array}$ & $\begin{array}{c}0.129 \\
(0.335)\end{array}$ & $\begin{array}{c}0.094 \\
(0.292)\end{array}$ & $\begin{array}{c}0.142 \\
(0.349)\end{array}$ & $\begin{array}{c}0.094 \\
(0.292)\end{array}$ & $\begin{array}{l}0.120 \\
(0.325)\end{array}$ & $\begin{array}{l}0.065 \\
(0.246)\end{array}$ \\
\hline College completion & $\begin{array}{l}0.085 \\
(0.280)\end{array}$ & $\begin{array}{c}0.094 \\
(0.292)\end{array}$ & $\begin{array}{l}0.096 \\
(0.295)\end{array}$ & $\begin{array}{c}0.091 \\
(0.288)\end{array}$ & $\begin{array}{l}0.062 \\
(0.242)\end{array}$ & $\begin{array}{l}0.104 \\
(0.305)\end{array}$ & $\begin{array}{c}0.071 \\
(0.257)\end{array}$ & $\begin{array}{l}0.098 \\
(0.297)\end{array}$ & $\begin{array}{l}0.103 \\
(0.304)\end{array}$ & $\begin{array}{l}0.068 \\
(0.246)\end{array}$ \\
\hline Married @ child born & $\begin{array}{c}0.864 \\
(0.343)\end{array}$ & $\begin{array}{c}0.934 \\
(0.248)\end{array}$ & $\begin{array}{l}0.858 \\
(0.349)\end{array}$ & $\begin{array}{l}0.935 \\
(0.247)\end{array}$ & $\begin{array}{c}0.838 \\
(0.369)\end{array}$ & $\begin{array}{c}0.951 \\
(0.215)\end{array}$ & $\begin{array}{c}0.674 \\
(0.469)\end{array}$ & $\begin{array}{c}0.984 \\
(0.125)\end{array}$ & $\begin{array}{c}0.894 \\
(0.308)\end{array}$ & $\begin{array}{l}0.983 \\
(0.128)\end{array}$ \\
\hline $\begin{array}{l}\text { Work in manufacturing } \\
\text { @ child born }\end{array}$ & $\begin{array}{c}0.053 \\
(0.225)\end{array}$ & $\begin{array}{c}0.040 \\
(0.197)\end{array}$ & $\begin{array}{c}0.050 \\
(0.218)\end{array}$ & $\begin{array}{c}0.041 \\
(0.199)\end{array}$ & $\begin{array}{c}0.037 \\
(0.190)\end{array}$ & $\begin{array}{c}0.045 \\
(0.208)\end{array}$ & $\begin{array}{c}0.038 \\
(0.191)\end{array}$ & $\begin{array}{c}0.044 \\
(0.206)\end{array}$ & $\begin{array}{c}0.039 \\
(0.195)\end{array}$ & $\begin{array}{c}0.051 \\
(0.221)\end{array}$ \\
\hline$\Lambda$ & 2,420 & 8,962 & 2,357 & 9,025 & 3,153 & 8,229 & 2,241 & 9,141 & 7,935 & 3,447 \\
\hline
\end{tabular}


Table 2: Continued.

\section{Father's events}

Child variables

$\begin{array}{lcccccccccc}\text { High school completion } & 0.470 & 0.488 & 0.470 & 0.488 & 0.460 & 0.493 & 0.453 & 0.491 & 0.505 & 0.105 \\ \text { by age } 19 & (0.499) & (0.500) & (0.499) & (0.500) & (0.498) & (0.500) & (0.498) & (0.500) & (0.500) & (0.306)\end{array}$

\section{Father variables}

High school completion

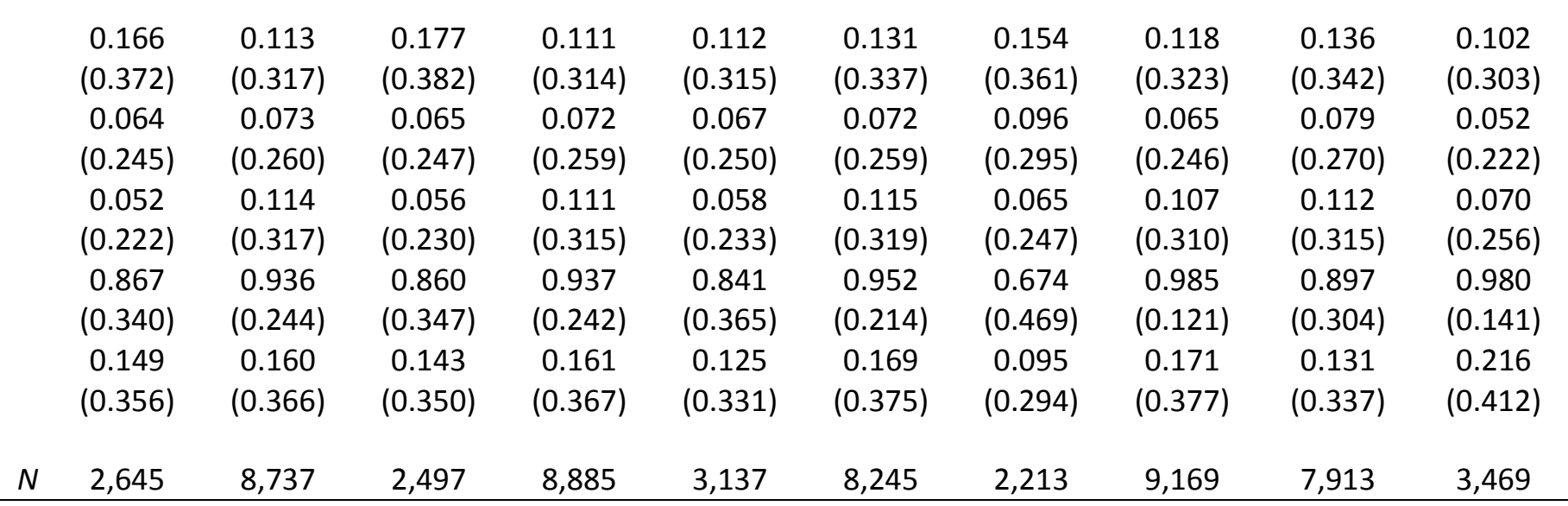

Note: Children who were at least 19 years old in 2011 were included in the sample. 


\begin{tabular}{lccc}
\hline Table 3: Prevalence of events (parents/mothers/fathers) & & \\
\hline & $\begin{array}{c}\text { Either/Both } \\
\text { Parents }\end{array}$ & Mother & Father \\
\cline { 2 - 4 } & & & \\
Single events & $34.05 \%$ & $21.26 \%$ & $23.24 \%$ \\
$\quad$ Lost employment & $32.82 \%$ & $20.71 \%$ & $21.94 \%$ \\
$\quad$ Gained employment & $28.21 \%$ & $27.70 \%$ & $27.56 \%$ \\
Lost partner & $23.59 \%$ & $19.69 \%$ & $19.44 \%$ \\
Gained partner & $70.86 \%$ & $69.72 \%$ & $69.52 \%$ \\
Moved & & & \\
Event combinations & $16.89 \%$ & $9.59 \%$ & $11.09 \%$ \\
Lost employment + Moved & $15.85 \%$ & $9.01 \%$ & $10.34 \%$ \\
Gained employment + Moved & $21.26 \%$ & $17.98 \%$ & $18.83 \%$ \\
Lost partner + Moved & $18.49 \%$ & $14.58 \%$ & $14.63 \%$ \\
$\quad$ Gained partner + Moved & $4.31 \%$ & $2.13 \%$ & $2.50 \%$ \\
Gained employment + Gained partner & $3.98 \%$ & $2.06 \%$ & $2.49 \%$ \\
Gained employment + Lost partner & $5.17 \%$ & $2.46 \%$ & $3.29 \%$ \\
Lost employment + Lost partner & $3.81 \%$ & $1.70 \%$ & $2.25 \%$ \\
$\quad$ Lost employment + Gained partner & &
\end{tabular}

Notes: Variables only included events that occurred when child was 1-17 years old. Children who were at least 19 years old in 2011 were included in the sample. $(\mathrm{N}=11,382)$ 


\begin{tabular}{|c|c|c|c|c|c|c|c|c|c|c|}
\hline \multirow{2}{*}{ Child variables } & \multicolumn{2}{|c|}{$\begin{array}{l}\text { Employment } \\
\text { loss }\end{array}$} & \multicolumn{2}{|c|}{$\begin{array}{l}\text { Employment } \\
\text { gain }\end{array}$} & \multicolumn{2}{|c|}{ Partner loss } & \multicolumn{2}{|c|}{ Partner gain } & \multicolumn{2}{|c|}{ Moved } \\
\hline & & & & & & & & & & \\
\hline Male $(0 / 1)$ & 0.003 & & 0.004 & & -0.001 & & 0.004 & & 0.004 & \\
\hline Black (0/1) & 0.107 & $* * *$ & 0.103 & $* * *$ & 0.080 & $* * *$ & 0.036 & $* * *$ & 0.053 & $* * *$ \\
\hline Other race $(0 / 1)$ & 0.047 & $* *$ & 0.066 & $* * *$ & 0.062 & $* * *$ & 0.065 & $* * *$ & 0.084 & $* * *$ \\
\hline Age in 2011 & -0.013 & $* * *$ & -0.014 & $* * *$ & -0.011 & $* * *$ & -0.008 & $* * *$ & -0.013 & $* * *$ \\
\hline Born in South & -0.031 & $* *$ & -0.022 & $*$ & 0.011 & & 0.003 & & 0.030 & $* *$ \\
\hline \multicolumn{11}{|l|}{ Mother variables } \\
\hline High school completion & 0.034 & $*$ & 0.031 & $*$ & -0.026 & $*$ & -0.008 & & 0.006 & \\
\hline Some college & 0.048 & $* *$ & 0.067 & $* * *$ & 0.026 & + & 0.016 & & 0.028 & $*$ \\
\hline College completion & -0.035 & $\dagger$ & -0.018 & & -0.087 & $* * *$ & -0.067 & $* * *$ & -0.018 & \\
\hline Married@ child born & 0.073 & & -0.033 & & -0.425 & $* *$ & -0.374 & $* *$ & -0.252 & $*$ \\
\hline Work in manu. @ child born & 0.051 & $*$ & 0.060 & $* *$ & -0.014 & & -0.013 & & 0.014 & \\
\hline \multicolumn{11}{|l|}{ Father variables } \\
\hline High school completion & -0.021 & & -0.012 & & -0.118 & $* * *$ & -0.046 & $* * *$ & -0.021 & \\
\hline Some college & -0.072 & $* * *$ & -0.061 & $* *$ & -0.097 & $* * *$ & -0.016 & & 0.016 & \\
\hline College completion & -0.133 & $* * *$ & -0.123 & $* * *$ & -0.153 & $* * *$ & -0.092 & $* * *$ & 0.051 & $* *$ \\
\hline Married @ child born & -0.187 & & -0.088 & & 0.208 & & -0.243 & $*$ & 0.113 & \\
\hline Work in manu. @ child born & 0.029 & $*$ & 0.022 & $\dagger$ & 0.023 & + & -0.009 & & -0.041 & $* * *$ \\
\hline Constant & 0.933 & $* * *$ & 0.945 & $* * *$ & 0.916 & $* * *$ & 1.146 & $* * *$ & 1.322 & $* * *$ \\
\hline F-test & 100.3 & & 115 & & 100. & & 213 & & 131. & \\
\hline$P>\chi^{2}$ & 0.00 & & 0.00 & & 0.0 & & 0.00 & & 0.00 & \\
\hline Adjusted $R^{2}$ & 0.13 & & 0.14 & & 0.13 & & 0.24 & & 0.16 & \\
\hline$n$ & & & & & 9,93 & & & & & \\
\hline
\end{tabular}

Notes: Variables only included events that occurred when child was 1-17 years old. Children who were at least 19 years old in 2011 were included in the sample. Results are log-odds.

$+\mathrm{p}<.10^{*} \mathrm{p}<.05 * * \mathrm{p}<.01 * * * \mathrm{p}<.001$ (two-tailed tests) 
parents who experienced events; this result underscores growth trends in employment and marital instability over time. Respondents born in the South were slightly less likely to have parents who experienced employment events and slightly more likely to experience residential moves during childhood. Running separate models for mothers' and fathers' events (results available upon request) indicated that higher levels of parental educational attainment appeared protective against employment and marital events for both fathers and mothers, whereas completing college increased the odds of experiencing a residential move. Working in manufacturing increased the chances of experiencing an employment or marital disruption for both mothers and fathers; evidence was mixed on its effect on marital change. Fathers who worked in manufacturing had a lower chance of residential moves. Though none of these results were surprising, it was important to consider these factors as a backdrop when thinking about children's educational outcomes. Accordingly, subsequent analyses of disruptive events controlled for covariates such as child's race, child's age in 2011, child born in the South, mother's and father's education, mother's and father's marital status at child's birth, and mother's and father's job in manufacturing.

\section{Analysis of events, event combinations and child outcomes}

The next step was to investigate how single events were related to child outcomes. Log-odds outcomes of logistic regression models testing the effects of parental disruptive events on children's educational outcomes are shown in table 5 . Single events did not have much impact on children's chances of high school completion, except for losing a partner, before parents' covariates were added to the model. However, all events led to lowered odds of college attendance and completion with the exception of the effect of moving on college attendance, which was null when controlled for child and parent characteristics. Appendices $C$ and D break out these results for mother and father events, instead of a combined parental event variable. An interesting difference to note here is that employment events for mothers did not appear to significantly affect the odds of any educational outcome for children once child, mother, and father factors are controlled, with the exception of a slight negative effect of employment gain on college attendance, whereas employment events for fathers showed significant negative effects on child college attendance and completion.

Combining events within a short timeframe led to worse educational outcomes. Almost all event combinations led to lowered chances of high school completion, college attendance and college completion; for parents' events, the exceptions were losing or gaining a partner with moving (and high school completion) and gaining employment with losing a partner (and college completion), for which results were insignificant. Notable again here is that event combinations with employment events led to worse outcomes when events occurred for fathers rather than mothers (see appendices $C$ and $D$ ).

In all cases, experiencing two events in the same two-year period was worse than experiencing one event. However, the degree of impact did not appear to fit the additive hypothesis. It is important to note that among those who experienced a single event could be those who experienced any number of events; among those who did not experience two events were those who experienced one event or perhaps the same two events but not within the same time period. In other words, comparison groups may not have been mutually exclusive, so comparing outcomes for single versus combined events was a complex undertaking.

\section{Analysis of events, event counts and child outcomes}

To look more simply at the number of events experienced and the interaction of their impacts in relation to the additive, amplification, and cumulation hypotheses, I created a variable for count of events, in which occurrences of all five events during childhood years were added together to create a simple index of instability. Few respondents experienced more than 20 events, therefore any count above 20 was coded as 20 events. Table 6 shows that events were largely detrimental to children's educational outcomes. For high school completion and college attendance, a model including a squared term for event counts was the best fit; once the event count surpassed three events, effects 
Table 5: Effects of single and combined events on children's educational outcomes, events for parents

\begin{tabular}{|c|c|c|c|c|c|c|c|c|c|c|c|c|}
\hline \multirow[b]{3}{*}{ Single events } & \multicolumn{4}{|c|}{$\begin{array}{c}\text { Completed high school } \\
\text { by age } 19\end{array}$} & \multicolumn{4}{|c|}{$\begin{array}{l}\text { Attended college } \\
\text { by age } 21\end{array}$} & \multicolumn{4}{|c|}{$\begin{array}{c}\text { College completion } \\
\text { by age } 25\end{array}$} \\
\hline & \multicolumn{2}{|c|}{$\begin{array}{l}\text { With child } \\
\text { covariates }\end{array}$} & \multicolumn{2}{|c|}{$\begin{array}{l}\text { With child, } \\
\text { mother \& } \\
\text { father } \\
\text { covariates }\end{array}$} & \multicolumn{2}{|c|}{$\begin{array}{l}\text { With child } \\
\text { covariates }\end{array}$} & \multicolumn{2}{|c|}{$\begin{array}{c}\text { With child, } \\
\text { mother \& } \\
\text { father } \\
\text { covariates }\end{array}$} & \multicolumn{2}{|c|}{$\begin{array}{l}\text { With child } \\
\text { covariates }\end{array}$} & \multicolumn{2}{|c|}{$\begin{array}{l}\text { With child, } \\
\text { mother \& } \\
\text { father } \\
\text { covariates }\end{array}$} \\
\hline & & & & & & & & & & & & \\
\hline Lost employment & 0.042 & & 0.052 & & -0.200 & $* * *$ & -0.145 & $* *$ & -0.324 & $* * *$ & -0.149 & $*$ \\
\hline Gained employment & 0.044 & & 0.006 & & -0.199 & $* * *$ & -0.193 & $* *$ & -0.243 & $* * *$ & -0.118 & \\
\hline Lost partner & -0.104 & $*$ & 0.031 & & -0.399 & $* * *$ & -0.203 & $* * *$ & -0.565 & $* * *$ & -0.256 & $* *$ \\
\hline Gained partner & -0.056 & & 0.016 & & -0.370 & $* * *$ & -0.247 & $* * *$ & -0.530 & $* * *$ & -0.289 & $* *$ \\
\hline Moved & 0.445 & $* * *$ & 0.445 & $* * *$ & 0.176 & $* *$ & 0.097 & & -0.071 & & -0.251 & $* *$ \\
\hline \multicolumn{13}{|l|}{ Event combinations } \\
\hline Lost employment + Moved & -0.254 & $* * *$ & -0.266 & $* * *$ & -0.370 & $* * *$ & -0.302 & $* * *$ & -0.539 & $* * *$ & -0.364 & $* * *$ \\
\hline Gained employment + Moved & -0.204 & $* * *$ & -0.216 & $* * *$ & -0.464 & $* * *$ & -0.434 & $* * *$ & -0.551 & $* * *$ & -0.421 & $* * *$ \\
\hline Lost partner + Moved & -0.141 & $* *$ & -0.061 & & -0.430 & $* * *$ & -0.294 & $* * *$ & -0.605 & $* * *$ & -0.364 & $* * *$ \\
\hline Gained partner + Moved & -0.071 & & -0.001 & & -0.383 & $* * *$ & -0.272 & $* * *$ & -0.490 & $* * *$ & -0.274 & $* *$ \\
\hline Gained employment + Gained partner & -0.274 & $* *$ & -0.186 & $\dagger$ & -0.423 & $* * *$ & -0.229 & $\dagger$ & -0.839 & $* * *$ & -0.485 & $*$ \\
\hline Gained employment + Lost partner & -0.276 & $* *$ & -0.224 & * & -0.706 & $* * *$ & -0.534 & $* * *$ & -0.474 & $*$ & -0.089 & \\
\hline Lost employment + Lost partner & -0.487 & $* * *$ & -0.392 & $* * *$ & -0.780 & $* * *$ & -0.585 & $* * *$ & -0.715 & $* * *$ & -0.375 & $*$ \\
\hline Lost employment + Gained partner & -0.304 & $* *$ & -0.239 & $*$ & -0.443 & $* * *$ & -0.226 & + & -0.774 & $* * *$ & -0.394 & $\dagger$ \\
\hline \multicolumn{13}{|c|}{$\begin{array}{l}\text { Notes: Each coefficient represents a separate model; coefficients are reported as log-odds. Only events that occurred when the child was } \\
\text { between } 1 \text { and } 17 \text { years old were included. Children must be at least } 19 \text { years old by } 2011 \text { to be included in the sample }(\mathrm{N}=11,382) \text {. For } \\
\text { event combinations, the order of events was unknown, and the two events occurred within the same two-year period. All models included } \\
\text { controls for child's sex, child's race, child's age in 2011, child born in the South, mother's and father's education, mother's and father's } \\
\text { marital status at child's birth, and mother's and father's job in manufacturing. } \\
+p<.10 * p<.05 * * p<.01 * * * p<.001 \text { (two-tailed tests) }\end{array}$} \\
\hline
\end{tabular}




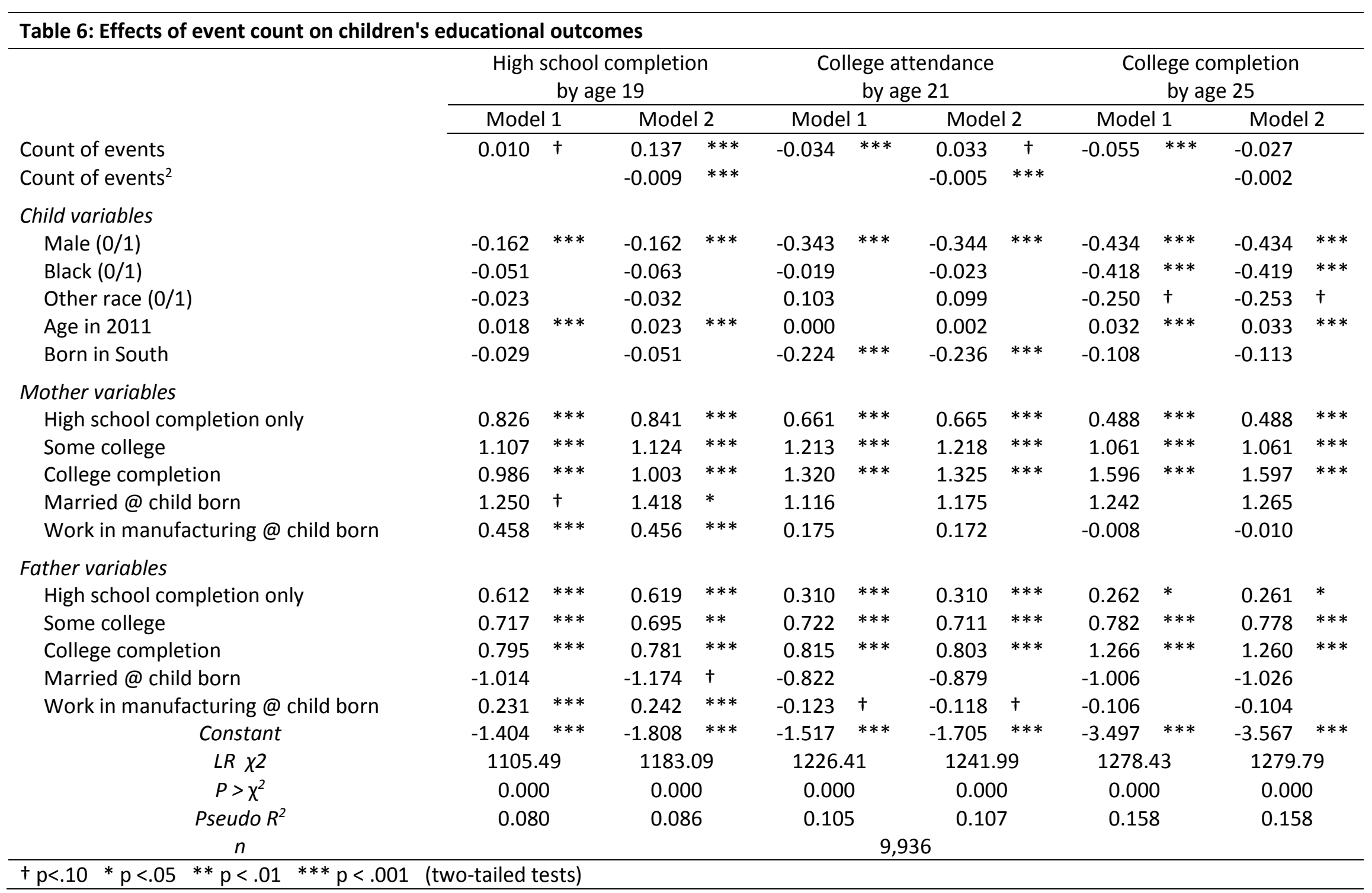


were increasingly negative. For college completion, the squared term was unnecessary and insignificant, and rather any number of events had a negative effect on child outcomes, with each additional event decreasing the odds of completing college. Though this was an admittedly general way of approaching the question of instability, it did illustrate that these events functioned disruptively in terms of their effects on children. It also underscored the finding that experiencing a larger number of events was worse than experiencing a single event.

\section{Managing overlapping event experiences}

One complication when comparing outcomes between single and combined events was that the comparison groups overlap. In other words, those who experienced a single event in one two-year period may also have experienced additional events in a different time; also, those who did not experience a combination of events could either have experienced no events or a single event. To test the outcomes for mutually exclusive categories, it was necessary to look within each two-year period separately and to analyse mothers' and fathers' events separately as well. A categorical variable was created with the categories being no events (reference indicator), both events $A$ and $B$ both occurred, only event A occurred, and only event B occurred. I limited this analysis to event combinations including a residential move, as these categories had large enough samples within each category to use biannual categorical indicators. Then, the average outcome across all years could be examined to see if event combinations generally led to worse outcomes.

Table 7 shows results from models using these categorical variables. Since these were biannual variables, the results were an average across all years. Models included year fixed effects. For all combinations, experiencing both events significantly negatively affected educational outcomes; with the slight exception of gaining a partner and moving for fathers, in all other cases the combination of events was a lot worse for children compared to single events. It was clear from the results that, on average, event combinations led to worse log-odds of attaining the outcome compared to single events. This mirrored earlier findings; having mutually exclusive comparison groups did not alter these conclusions.

\section{Discussion}

I conducted this analysis by investigating if and to what extent parental disruptions are associated with negative impacts on children's educational attainment. I began by asking the question: How often are events found in combination with one another? Combinations of disruptive events were surprisingly frequent, particularly when considering the proportion of parents who experienced additional events given that they already experienced one disruptive event. This meant that looking at the impacts of just one event may have masked additional negative associations or incorrectly assigned negative impacts to one disruptive event rather than another. I also asked: Which combinations are most common (i.e. which events are often experienced in the same family within a short time frame? It was clearly most common to combine a residential move with either an employment or a partnership change. Relationship and employment status changes did not combine as often.

I next asked the question: Do certain event combinations lead to lower educational outcomes for children than others? It was difficult to compare across sets of events, and combinations of events seemed generally within the same range of effect size. Combinations clearly mattered, however, and across model specifications, parents' experience of two contemporaneous events led to a lower probability of their children attaining all considered educational outcomes compared to parents' experience of single events.

Do any of these combinations of events lead to positive educational outcomes for children? The educational outcomes following disruptive events were overwhelmingly negative, if not insignificant. Combinations of events were negative for all outcomes as well, whereas single events had little effect on high school completion.

Is a higher count of events worse (in terms of children's educational outcomes) than a lower count? My results showed that this was indeed the case. Each disruptive event came at an incrementally negative cost to children's educational attainment when events occurred in combination generally across childhood. A caveat on this finding was that the number of events parents experienced while their 
Table 7: Effects of single and combined events on children's educational attainment

\begin{tabular}{|c|c|c|c|c|c|c|c|c|c|c|c|c|}
\hline \multirow[b]{3}{*}{ Employment lost \& Move } & \multicolumn{4}{|c|}{$\begin{array}{c}\text { High school completion } \\
\text { by age } 19\end{array}$} & \multicolumn{4}{|c|}{$\begin{array}{c}\text { College attendance } \\
\text { by age } 21\end{array}$} & \multicolumn{4}{|c|}{$\begin{array}{c}\text { College completion } \\
\text { by age } 25\end{array}$} \\
\hline & \multicolumn{2}{|c|}{ Mothers } & \multicolumn{2}{|c|}{ Fathers } & \multicolumn{2}{|c|}{ Mothers } & \multicolumn{2}{|c|}{ Fathers } & \multicolumn{2}{|c|}{ Mothers } & \multicolumn{2}{|c|}{ Fathers } \\
\hline & -0.289 & $* * *$ & -0.304 & $* * *$ & -0.320 & $* * *$ & -0.287 & $* * *$ & -0.581 & $* * *$ & -0.616 & $* * *$ \\
\hline Move & 0.185 & $* * *$ & 0.105 & $* * *$ & 0.017 & & 0.004 & & -0.248 & $* * *$ & -0.214 & $* * *$ \\
\hline Employ & 0.162 & $* *$ & 0.119 & $*$ & 0.008 & & -0.059 & & -0.066 & & -0.251 & $* *$ \\
\hline Emp & -0.169 & $* *$ & -0.324 & $* * *$ & -0.337 & $* * *$ & -0.349 & $* * *$ & -0.589 & $* * *$ & -0.551 & $* * *$ \\
\hline Move & 0.171 & $* * *$ & 0.104 & $* * *$ & 0.015 & & 0.006 & & -0.250 & $* * *$ & -0.219 & $* * *$ \\
\hline Employ & 0.037 & & 0.046 & & -0.093 & & -0.102 & $\dagger$ & -0.134 & $\dagger$ & -0.248 & $* *$ \\
\hline Lost & -0.232 & $* * *$ & -0.168 & $* * *$ & -0.377 & $* * *$ & -0.299 & $* * *$ & -0.579 & $* * *$ & -0.552 & $* * *$ \\
\hline Mov & 31 & $* * *$ & 0.065 & $* * *$ & -0.037 & $\dagger$ & -0.009 & & -0.269 & $* * *$ & -0.230 & $* *$ \\
\hline Lost & 5 & $* * *$ & 0.175 & $*$ & 31 & $\dagger$ & 0.071 & & -0.268 & $*$ & -0.145 & \\
\hline Gai & $-U$. & $*$ & & $* *$ & & $* * *$ & 72 & 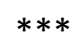 & & $* * *$ & -0.422 & $* * *$ \\
\hline Mov & 0.057 & $* *$ & 0.053 & $* *$ & -0.052 & $* *$ & -0.022 & & -0.280 & $* * *$ & -0.249 & $* * *$ \\
\hline Gain & -0.064 & & -0.017 & & -0.146 & & -0.136 & & -0.490 & $* *$ & -0.495 & $* *$ \\
\hline $\begin{array}{l}\text { period. All models include } \\
\text { mother's and father's edu } \\
\text { in manufacturing. } \\
+\mathrm{p}<.10 * \mathrm{p}<.05 * * \mathrm{p}<\end{array}$ & $1 \quad * * *$ & 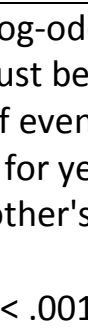 & gevo & 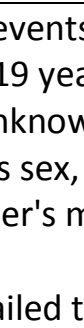 & S) & 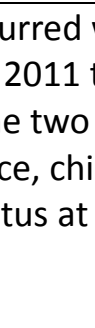 & $=0 \pi$ & 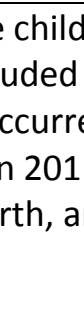 & 政 & 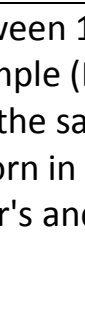 & 8 & 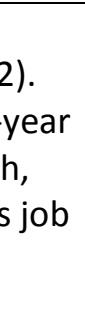 \\
\hline
\end{tabular}


children were between the ages of 1 and 17 years varied between 0 and 20, though the models controlled for likely factors (race, education, married when child was born, etc.) to address relevant selection. While $50 \%$ of children had parents who experienced one or two events during their childhood, an additional $40 \%$ of children had parents who experienced between three and ten events during their childhood. So, though experiencing 15 or more events was less likely, the majority of the sample experienced a high count of disruptions.

The data showed that experiencing multiple events was not rare across the life course. Therefore, the fact that additional events were associated with increasingly negative outcomes is worrying for children's educational attainment. It also points to a potential group of increasingly disadvantaged families. There was no consistent additive pattern to support an additive hypothesis; however, the outcomes of combined events were consistently worse than single events, indicating that the amplification hypothesis provided a solid explanation. In other words, associations of single events could not be simply added together to provide the association for multiple events, but the outcome of two events combined was more strongly negative than the effect of one of those events alone. For example, residential moves were indeed associated with poorer educational outcomes. When a move in residence was combined with an event such as a parent's divorce, the educational outcomes were worse than for the move by itself. These outcomes suggested that the effects of single events in fact overlap, in which some part of the association of a divorce looked at in isolation was actually the association of a divorce combined with a residential move, and vice versa.

The cumulation hypothesis focused on the count of events more generally to see if effects tapered off. Looking at a count of all events found that, for high school completion and college attendance, effects were indeed non-linear. For college completion, a linear model was a better choice. Rather than associations tapering off, however, they worsened as the count increased. Even when including controls for child and parent characteristics, more instability owing to the increase in disruptive events led to worse educational outcomes for children.
I offer several cautions on these findings. It is possible that events occurring the same year were not linked in a "cascade"; I had no way of determining reasons for events occurring within close timing of each other in the PSID. Future work should delve further into the reasons that these events might combine. Additionally, there were other disruptive events that could easily combine with the events on which these analyses focused (e.g. health-related events); those other disruptive events should be analysed in the future.

When thinking about the consequences for policy specifically relating to families and children, it is important to remember how different the processes, decisions and costs of attaining a high school diploma are compared to college attendance and college graduation. High school is free and compulsory, whereas college can be quite expensive and is more choice-based. Therefore, the differences in effects on the probability of high school graduation as compared to college attendance and especially college completion were not surprising. Both single events and event combinations seemed to matter more for college outcomes compared to high school completion, with event combinations leading to an even lower probability of attaining college outcomes.

There are several interesting immediate policy implications here. First, because disruptive events often combine, and because the experience of these combinations led to lower educational attainment (compared to the experience of single disruptive events), perhaps policies should first focus on stopping the cascade of disruptive events and then work on mending the consequences of events that have already occurred. Policies that allow people to remain living in their rented or mortgaged homes following a job loss are a great example of this sort of policy intervention. Second, there were some differences between mothers' and fathers' experiences of disruptive events, but largely the effects on children looked quite similar, and policies should address this. Third, residential moves and partner losses showed the largest effects on children's educational attainment. Educational policy makers should be aware of the negative consequences that children face when they move. 
Additionally, partner losses and gains figured heavily in college graduation, findings of which financial aid officers as well as academic mentors should be aware.

\section{Acknowledgements}

I thank Jennie Brand, Robert Mare, Judith Seltzer, Michael Seltzer, Jason Beckfield, Lisa Berkman, and Ben Jarvis for helpful comments and suggestions. This research was supported by a National Institute on Aging training grant at the California Center for Population Research (5T32AG033533) and an advanced quantitative methodology training grant (R305B080016) awarded by the Institute of Education Sciences of the U.S. Department of Education, both at the University of California, Los Angeles.

\section{References}

Adams, G., \& Dubay, L. (2014). Exploring Instability and Children's Wellbeing: Insights from a Dialogue Among Practitioners, Policymakers, and Researchers. Washington, DC: The Urban Institute.

Amato, P. R. (2000). The Consequences of Divorce for Adults and Children. Journal of Marriage and Family, 62(4):1269-1287. https://doi.org/10.1111/j.1741-3737.2000.01269.x

Amato, P. R., \& Booth, A. (1991). Consequences of Parental Divorce and Marital Unhappiness for Adult WellBeing. Social Forces, 69(3):895-914. https://doi.org/10.1093/sf/69.3.895

Amato, P. R., \& Keith, B. (1991). Parental Divorce and the Well-Being of Children: A Meta-Analysis. Psychological Bulletin, 110(1):26-46. https://doi.org/10.1037/0033-2909.110.1.26

Beller, E. (2009). Bringing Intergenerational Social Mobility Research into the Twenty-first Century: Why Mothers Matter. American Sociological Review, 74:507-528. https://doi.org/10.1177/000312240907400401

Borgen, L., \& Rumbaut, R. G. (2011). Coming of Age in "America's Finest City": Transitions to Adulthood Among Children of Immigrants in San Diego. In M. C. Waters, P. J. Carr, M. J. Kefalas, J. Holdaway (Eds.), Coming of Age in America: The Transition to Adulthood in the Twenty-First Century. Oakland, CA: University of California Press. https://doi.org/10.1525/california/9780520270923.003.0005

Brand, J., \& Simon Thomas, J. (2014). Job Displacement Among Single Mothers: Effects on Children's Outcomes in Young Adulthood. American Journal of Sociology, 119(4):955-1001. https://doi.org/10.1086/675409

Brown, S. L. (2010). Marriage and Child Well-Being: Research and Policy Perspectives. Journal of Marriage and Family, 72:1059-1077. https://doi.org/10.1111/j.1741-3737.2010.00750.x

Buchmann, M. (1989). The Script of Life in Modern Society: Entry into Adulthood in a Changing World. Chicago: The University of Chicago Press.

Burdick-Will, J., Ludwig, J., Raudenbush, S.W., Sampson, R. J., Sanbonmatsu, L., \& Sharkey, P. (2011). Converging Evidence for Neighborhood Effects on Children's Test Scores: An Experimental, Quasi-Experimental, and Observational Comparison. In G.D. Duncan \& R. J. Murnane (Eds.), Whither Opportunity? Rising Inequality, Schools, and Children's Life Chances (pp. 255-276). Chicago: Spencer Foundation; New York: Russell Sage Foundation.

Cavanagh, S., \& Fomby, P. (2011). Family Instability, School Context, and the Academic Careers of Adolescents. Sociology of Education, 85(3):81-97.

Cavanagh, S., \& Huston, A. (2006). Family Instability and Children's Early Problem Behavior. Social Forces, 85(1):551-581. https://doi.org/10.1353/sof.2006.0120

Charles, K. K., \& Stephens Jr, M. (2004). Job Displacement, Disability, and Divorce. Journal of Labor Economics, 22:489-522. https://doi.org/10.1086/381258

Clark, W. A.V. (2016). Life Events and Moves Under Duress: Disruption in the Life Course and Mobility Outcomes. Longitudinal and Life Course Studies 7(3): 218-239. https://doi.org/10.14301/llcs.v7i3.376 
Cooper, C. E., Osborne, C.A., Beck, A.N., \& McLanahan, S. S. (2011). Partnership Instability, School Readiness, and Gender Disparities. Sociology of Education, 84(3):246-259. https://doi.org/10.1177/0038040711402361

Corak, M. (2004). Generational income mobility in North America and Europe: an introduction. In M. Corak (Ed.), Generational income mobility in North America and Europe (pp. 1-37). Cambridge: Cambridge University Press. https://doi.org/10.1017/CBO9780511492549

Crosnoe, R., Chambers Prickett, K., Smith, C., \& Cavanagh, S. (2014). Changes in Young Children's Family Structures and Child Care Arrangements. Demography, 51:459-483. https://doi.org/10.1007/s13524013-0258-5

Davis, S. J., \& M. von Wachter, T. (2011). Recessions and the Cost of Job Loss. NBER Working Paper 17638. https://doi.org/10.3386/w17638

DeWit, D. J. (1998). Frequent Childhood Geographic Relocation: Its Impact on Drug Use Initiation and the Development of Alcohol and Other Drug-Related Problems Among Adolescents and Young Adults. Addictive Behaviors, 23(5):623-634. https://doi.org/10.1016/S0306-4603(98)00023-9

DiPrete, T., \& McManus, P. (2000). Family Change, Employment Transitions, and the Welfare State: Household Income Dynamics in the United States and Germany. American Sociological Review, 65:343-370. https://doi.org/10.2307/2657461

Elder, G. H., Jr. (1999)[1974]. Children of the Great Depression: Social Change in Life Experience (25 ${ }^{\text {th }}$ Anniversary Edition). Boulder, CO: Westview Press.

Elder, G. H., Jr., Kirkpatrick Johnson, M., \& Crosnoe, R. (2004). The Emergence and Development of Life Course Theory. In J. T. Mortimer and M. J. Shanahan (Eds.), Handbook of the Life Course (pp. 3-19). New York, NY: Springer Publishing.

Falaris, E. M., \& Peters, H. E. (1998). Survey Attrition and Schooling Choices. The Journal of Human Resources, 33(2):531-554. https://doi.org/10.2307/146440

Fitzgerald, J. M. (2011). Attrition in Models of Intergenerational Links Using the PSID with Extensions to Health and to Sibling Models. The B.E. Journal of Economic Analysis \& Policy, 11:3(Advances), Article 2.

Fomby, P. (2013). Family Instability and College Enrollment and Completion. Population Research and Policy Review, 32:469-494. https://doi.org/10.1007/s11113-013-9284-7

Fomby, P., \& Bosick, S. J. (2013). Family Instability and the Transition to Adulthood. Journal of Marriage and Family, 75:1266-1287. https://doi.org/10.1111/jomf.12063

Fomby, P., and Sennott, C. A. (2013). Family Structure Instability and Mobility: The Consequences for Adolescents' Problem Behavior. Social Science Research, 42:181-206. https://doi.org/10.1016/j.ssresearch.2012.08.016

Furstenberg, F. F. (1995). Family Change and the Welfare of Children: What Do We Know and What Can We Do About It? In K. Oppenheim Mason and A-M. Jensen (Eds.), Gender and Family Change in Industrialized Countries (pp. 247-257). Oxford: Clarendon Press.

Garfinkel, I., \& McLanahan, S. (1986). Single Mothers and Their Children. Washington, DC: The Urban Institute Press.

Gennetian, L. A., Wolf, S., Hill, H. D., \& Morris, P. A. (2015). Intrayear Household Income Dynamics and Adolescent School Behavior. Demography, 52:455-483. https://doi.org/10.1007/s13524-015-0370-9

Goldberg, J, \& Carlson, M. (2014). Parents' Relationship Quality and Children's Behavior in Stable Married and Cohabiting Families. Journal of Marriage and Family, 76(4):762-777. https://doi.org/10.1111/jomf.12120

Gupta, S, Smock, P. J. \& Manning, W. D. (2004). Moving Out: Transition to Nonresidence Among Resident Fathers in the United States, 1968-1997. Journal of Marriage and Family, 66:627-638. https://doi.org/10.1111/j.0022-2445.2004.00042.x

Hardy, B. L. (2014). Childhood Income Volatility and Adult Outcomes. Demography, 51:1641-1665. https://doi.org/10.1007/s13524-014-0329-2 
Hao, L. (1996). Family Structure, Private Transfers, and the Economic Well-Being of Families with Children. Social Forces, 75(1):269-292. https://doi.org/10.1093/sf/75.1.269

Hipple, S. Self-employment in the United States. Monthly Labor Review, September 2010.

Hofferth, S. L. (2006). Residential Father Type and Child Well-being: Investment Versus Selection. Demography, 43(1):53-77. https://doi.org/10.1353/dem.2006.0006

Holland, P. W. (1986). Statistics and Causal Inference. Journal of the American Statistical Association, 81:945970. https://doi.org/10.1080/01621459.1986.10478354

Hsin, A., \& Felfe, C. (2014). When Does Time Matter? Maternal Employment, Children's Time With Parents, and Child Development. Demography, 51:1867-1894. https://doi.org/10.1007/s13524-014-0334-5

Ingersoll, G. M., Scamman, G. P. \& Eckerling, W. D. (1989). Geographic Mobility and Student Achievement in an Urban Setting. Educational Evaluation and Policy Analysis, 11(2):143-149. https://doi.org/10.3102/01623737011002143

Isaacs, J. (2014). How Do We Help Children Thrive Amid Instability? The Urban Institute, online article, accessed September 1, 2014.

Jaffee, S. R., Moffitt, T.E., Caspi, A., \& Taylor, A. (2003). Life with (or without) Father: The Benefits of Living with Two Biological Parents Depend on the Father's Antisocial Behavior. Child Development, 74(1):109-126. https://doi.org/10.1111/1467-8624.t01-1-00524

Jelleyman, T., \& Spencer, N. (2008). Residential Mobility in Childhood and Health Outcomes: A Systematic Review. Journal of Epidemiology and Community Health, 62:584-592. https://doi.org/10.1136/jech.2007.060103

Kessler, R., Gillis-Light, J., Magee, W. G., Kendler, K.S. \& Eaves, L. J. (1997). Childhood Adversity and Adult Psychopathology. In I. H. Gotlib \& B. Wheaton (Eds.), Stress and Adversity over the Life Course: Trajectories and Turning Points (pp. 29-49). New York, NY: Cambridge University Press. https://doi.org/10.1017/СBO9780511527623.002

Kogan, I., Unt, M., \& Saar, E. (2007). Youth Unemployment in the Enlarged European Union. In S. Scherer, R. Pollak, G. Otte, and M. Gangl (Eds.), From Origin to Destination: Trends and Mechanisms in Social Stratification Research (pp. 182-213). Frankfurt/New York: Campus Verlag.

Malia, J. A. (2006). Basic Concepts and Models of Family Stress. Stress, Trauma, and Crisis, 9:3-4, 141-160. https://doi.org/10.1080/15434610600853717

Mayer, K. U. (2009). New Directions in Life Course Research. Annual Review of Sociology, 35:413-433. https://doi.org/10.1146/annurev.soc.34.040507.134619

McLanahan, S., \& Percheski, C. (2008). Family Structure and the Reproduction of Inequalities. Annual Review of Sociology, 34:257-76. https://doi.org/10.1146/annurev.soc.34.040507.134549

McLeod, J. D., \& Almazan, E. P. (2004). Connections between Childhood and Adulthood. In J. T. Mortimer and M. J. Shanahan (Eds.), Handbook of the Life Course (pp. 391-411). New York, NY: Springer Publishing.

Mitchell, C., McLanahan, S., Hobcraft, J., Brooks-Gunn, J., Garfinkel, I., \& Notterman, D. (2015). Family Structure Instability, Genetic Sensitivity, and Child Well-Being. American Journal of Sociology, 120(4): 1195-1225. https://doi.org/10.1086/680681

Morgan, S. L., \& Winship, C. (2007). Counterfactuals and Causal Inference: Methods and Principles for Social Research. New York, NY: Cambridge University Press. https://doi.org/10.1017/CBO9780511804564

Mortimer, J. T., Lei Zhang, F., Hussemann, J., \& Wu, C-Y. (2014). Parental Economic Hardship and Children's Achievement Orientations. Longitudinal and Life Course Studies, 5(2):105.

https://doi.org/10.14301/llcs.v5i2.271

Oreopoulos, P., Page, M., \& Huff Stevens, A. (2008). The Intergenerational Effects of Worker Displacement. Journal of Labor Economics, 6(3):455-483. https://doi.org/10.1086/588493

Osborne, C., \& McLanahan, S. 2007. Partnership Instability and Child Wellbeing. Journal of Marriage and Family, 69(4):1065-1083. https://doi.org/10.1111/j.1741-3737.2007.00431.x 
Panel Study of Income Dynamics, public use dataset. Produced and distributed by the Survey Research Center, Institute for Social Research, University of Michigan, Ann Arbor, MI (2017).

Parsons, S., Schoon, I., \& Vignoles, A. (2014. Parental Worklessness and Children's Early School Achievement and Progress. Longitudinal and Life Course Studies, 5(1):19-41. https://doi.org/10.14301/Ilcs.v5i1.230

Paul, K. I., \& Moser, K. (2009). Unemployment Impairs Mental Health: Meta-Analyses. Journal of Vocational Behavior, 74(3): 264-282. https://doi.org/10.1016/j.jvb.2009.01.001

Pearlin, L. I., Menaghan, E. G., Lieberman, M. A. \& Mullan, J. T. (1981). The Stress Process. The Journal of Health and Social Behavior, 22(4):337-356. https://doi.org/10.2307/2136676

Pearlin, L. I. (2010). The Life Course and the Stress Process: Some Conceptual Comparisons. Journal of Gerontology: Social Sciences, 65B(2):207-215. https://doi.org/10.1093/geronb/gbp106

Pettit, B., \& McLanahan, S. (2003). Residential Mobility and Children's Social Capital: Evidence from an Experiment. Social Science Quarterly, 84:632-649. https://doi.org/10.1111/1540-6237.8403009

Rhodes, W., \& Hoey, K. (1994). Overcoming Childhood Misfortune: Children Who Beat the Odds. Praeger Publishers: Westport, CT.

Riley, M. W., \& Riley, J. W. (1994). Age Integration and the Lives of Older People. The Gerontologist, 34(1):110115. https://doi.org/10.1093/geront/34.1.110

Rindfuss, R. R., Swicegood, C. G., \& Rosenfeld, R. A. (1987). Disorder in the Life Course: How Common and Does It Matter? American Sociological Review, 52(6): 785-801. https://doi.org/10.2307/2095835

Ryan, R., Claessens, A., \& Markowitz, A.J. (2013). Family structure and children's behavior. Focus, 30(2): 11-14.

Sanbonmatsu, L., Ludwig, J., Katz, L. F., Gennetian, L. A., Duncan, G. J., Kessler, R. C., Adam, E., McDade, T. W. \& Lindau, S. T. (2011). Moving to Opportunity for Fair Housing Demonstration Program: Final Impacts Evaluation. U.S. Department of Housing and Urban Development, Office of Policy Development \& Research.

Sayer, L. C., England, P., Allison, P. \& Kangas, N. (2011). She Left, He Left: How Employment and Satisfaction Affect Men's and Women's Decisions to Leave Marriages. American Journal of Sociology, 116(6):19822018. https://doi.org/10.1086/658173

Schoeni, R., \& Wiemers, E. (2015). The Implications of Selective Attrition for Estimates of Intergenerational Elasticity of Family Income. Journal of Economic Inequality, forthcoming. https://doi.org/10.1007/s10888-015-9297-z

Seltzer, J. (1994). Consequences of Marital Dissolution for Children. Annual Review of Sociology, 20:235-266. https://doi.org/10.1146/annurev.so.20.080194.001315

Shanahan, M. (2000). Pathways to Adulthood in Changing Societies: Variability and Mechanisms in Life Course Perspective. Annual Review of Sociology, 26:667-692. https://doi.org/10.1146/annurev.soc.26.1.667

Simon Thomas, J. Four Decades of Disruptive Events: Prevalence and Effects on Children's Educational Attainment. Manuscript available upon request.

Simon Thomas, J. Disruptive Events in Childhood and Children's Educational Attainment: Does Timing Matter? Manuscript available upon request.

South, S. J., Crowder, K.D., \& Trent, K. (1998). Children's Residential Mobility and Neighborhood Environment following Parental Divorce and Remarriage. Social Forces, 77 (2): 667-693. https://doi.org/10.1093/sf/77.2.667

South, S. J., Haynie, D. L. \& Bose, S. (2007). Student Mobility and School Dropout. Social Science Research, 36:68-94. https://doi.org/10.1016/j.ssresearch.2005.10.001

Stevens, A. H., \& Schaller, J. (2011). Short-Run Effects of Parental Job Loss on Children's Academic Achievement. Economic of Education Review, 30(2):289-299. https://doi.org/10.1016/j.econedurev.2010.10.002

Swartz, T. T., Hartmann, D., \& Mortimer, J. T. (2011). Transitions to Adulthood in the Land of Lake Wobegon. In M. C. Waters, P. J. Carr, M. J. Kefalas, \& J. Holdaway (Eds.), Coming of Age in America: The Transition to Adulthood in the Twenty-First Century. Berkeley, Los Angeles, London: University of California Press. https://doi.org/10.1525/california/9780520270923.003.0003 
Sweeney, M. (2010). Remarriage and Stepfamilies: Strategic Sites for Family Scholarship in the 21st Century. Journal of Marriage and Family, 72(3):667-684. https://doi.org/10.1111/j.1741-3737.2010.00724.x

Sweeney, M. (2011). Family-Structure Instability and Adolescent Educational Outcomes: A Focus on Families with Stepfathers. In G.D. Duncan and R. J. Murnane (Eds.), Whither Opportunity? Rising Inequality, Schools, and Children's Life Chances (pp. 229-252). Chicago: Spencer Foundation; New York: Russell Sage Foundation.

Tach, L., \& Eads, A. (2014). The cost of breaking up. Focus, 30(2):15-20.

Tach, L., \& Eads, A. (2015). Trends in the Economic Consequences of Marital and Cohabitation Dissolution in the United States. Demography, 52:401-432. https://doi.org/10.1007/s13524-015-0374-5

Thomas, J. R., \& Högnäs, R. S. (2015). The Effect of Parental Divorce on the Health of Adult Children. Longitudinal and Life Course Studies 6(3):279. https://doi.org/10.14301/llcs.v6i3.267

Uhlenberg, P., \& Mueller, M. (2004). Family Context and Individual Well-Being. In J. T. Mortimer \& M. J. Shanahan (Eds.), Handbook of the Life Course (pp. 123-148). New York, NY: Springer Publishing.

U.S. Department of Education, National Center for Education Statistics. (2014). The Condition of Education 2014 (NCES 2014-083), Educational Attainment.

Weitzman, L. J. (1985). "The Divorce Law Revolution and the Transformation of Legal Marriage." Pp. 301-348 in K. Davis with A. Grossbard-Shechtman (Eds.) Contemporary Marriage: Comparative Perspectives on a Changing Institution. New York: Russell Sage.

Werner, E. E., \& Smith, R. S. (1992). Overcoming the Odds: High Risk Children from Birth to Adulthood. Ithaca and London: Cornell University Press.

Wheaton, B., \& Gotlib, I. H. (1997). Trajectories and Turning Points: Concepts and Themes. In I. H. Gotlib \& B. Wheaton (Eds.), Stress and Adversity over the Life Course: Trajectories and Turning Points (pp. 1-25). New York, NY: Cambridge University Press. https://doi.org/10.1017/CBO9780511527623.001

Wu, L. L., \& Martinson, B. C. (1993). Family Structure and the Risk of a Premarital Birth. American Sociological Review, 58(2):210-232.

Wu, L. L. (1996). Effects of Family Instability, Income, and Income Instability on the Risk of a Premarital Birth. American Sociological Review, 61(3): 386-406. https://doi.org/10.2307/2096355

Young, C. (2012). Losing a Job: The Nonpecuniary Cost of Unemployment in the United States. Social Forces, 91(2):609-634. https://doi.org/10.1093/sf/sos071

\section{Endnotes}

1. I underscored the idea of disruption or shocks in children's lives here rather than the notion of parents both working or single mothers working. Recent research shows that it is not the amount but rather the quality of time spent with children that affects their wellbeing (Hsin \& Feife, 2014).

2. DiPrete and McManus (2000) include changing jobs with the same employer, changing employers but keeping a similar job, and changing partners as events. I eliminated these events because I believe that the shock of those events was likely to be more nuanced and more complex to disentangle for the effects on children. Those authors also include entry into self-employment, which I eliminated from my analysis due to low sample size (1.59\% for mothers and $1.33 \%$ for fathers across all survey years). 


\section{Appendix}

\begin{tabular}{|c|c|c|c|c|c|c|c|c|c|c|}
\hline \multicolumn{11}{|c|}{ Appendix A: Full Descriptive Statistics (events for mothers) } \\
\hline & \multicolumn{2}{|c|}{ Employment loss } & \multicolumn{2}{|c|}{ Employment gain } & \multicolumn{2}{|c|}{ Partner loss } & \multicolumn{2}{|c|}{ Partner gain } & \multicolumn{2}{|c|}{ Moved } \\
\hline & Yes & No & Yes & No & Yes & No & Yes & No & Yes & No \\
\hline & $\begin{array}{l}\text { Mean } \\
\text { (SD) }\end{array}$ & $\begin{array}{l}\text { Mean } \\
(S D)\end{array}$ & $\begin{array}{c}\text { Mean } \\
(S D)\end{array}$ & $\begin{array}{c}\text { Mean } \\
(S D)\end{array}$ & $\begin{array}{c}\text { Mean } \\
(S D)\end{array}$ & $\begin{array}{c}\text { Mean } \\
(S D)\end{array}$ & $\begin{array}{l}\text { Mean } \\
\text { (SD) }\end{array}$ & $\begin{array}{c}\text { Mean } \\
(S D)\end{array}$ & $\begin{array}{c}\text { Mean } \\
(S D)\end{array}$ & $\begin{array}{c}\text { Mean } \\
(S D)\end{array}$ \\
\hline \multicolumn{11}{|l|}{ Child variables } \\
\hline $\begin{array}{l}\text { High school completion } \\
\text { by age } 19\end{array}$ & $\begin{array}{c}0.489 \\
(0.500)\end{array}$ & $\begin{array}{c}0.483 \\
(0.500)\end{array}$ & $\begin{array}{c}0.488 \\
(0.500)\end{array}$ & $\begin{array}{c}0.483 \\
(0.500)\end{array}$ & $\begin{array}{c}0.460 \\
(0.498)\end{array}$ & $\begin{array}{c}0.493 \\
(0.500)\end{array}$ & $\begin{array}{l}0.458 \\
(0.498)\end{array}$ & $\begin{array}{c}0.490 \\
(0.500)\end{array}$ & $\begin{array}{c}0.507 \\
(0.500)\end{array}$ & $\begin{array}{c}0.432 \\
(0.495)\end{array}$ \\
\hline $\begin{array}{l}\text { College attendance } \\
\text { by age } 21\end{array}$ & $\begin{array}{c}0.249 \\
(0.433)\end{array}$ & $\begin{array}{c}0.261 \\
(0.439)\end{array}$ & $\begin{array}{c}0.242 \\
(0.428)\end{array}$ & $\begin{array}{c}0.263 \\
(0.440)\end{array}$ & $\begin{array}{c}0.214 \\
(0.410)\end{array}$ & $\begin{array}{c}0.276 \\
(0.447)\end{array}$ & $\begin{array}{c}0.211 \\
(0.408)\end{array}$ & $\begin{array}{c}0.271 \\
(0.444)\end{array}$ & $\begin{array}{c}0.269 \\
(0.444)\end{array}$ & $\begin{array}{l}0.235 \\
(0.424)\end{array}$ \\
\hline $\begin{array}{l}\text { College completion } \\
\text { by age } 25\end{array}$ & $\begin{array}{c}0.104 \\
(0.300)\end{array}$ & $\begin{array}{c}0.137 \\
(0.344)\end{array}$ & $\begin{array}{c}0.106 \\
(0.307)\end{array}$ & $\begin{array}{c}0.136 \\
(0.343)\end{array}$ & $\begin{array}{c}0.079 \\
(0.270)\end{array}$ & $\begin{array}{c}0.150 \\
(0.357)\end{array}$ & $\begin{array}{c}0.071 \\
(0.257)\end{array}$ & $\begin{array}{c}0.144 \\
(0.351)\end{array}$ & $\begin{array}{c}0.121 \\
(0.326)\end{array}$ & $\begin{array}{l}0.152 \\
(0.359)\end{array}$ \\
\hline Male $(0 / 1)$ & $\begin{array}{c}0.516 \\
(0.500)\end{array}$ & $\begin{array}{c}0.511 \\
(0.500)\end{array}$ & $\begin{array}{c}0.515 \\
(0.500)\end{array}$ & $\begin{array}{c}0.511 \\
(0.500)\end{array}$ & $\begin{array}{c}0.511 \\
(0.500)\end{array}$ & $\begin{array}{c}0.513 \\
(0.500)\end{array}$ & $\begin{array}{c}0.515 \\
(0.500)\end{array}$ & $\begin{array}{c}0.511 \\
(0.500)\end{array}$ & $\begin{array}{c}0.514 \\
(0.500)\end{array}$ & $\begin{array}{c}0.507 \\
(0.500)\end{array}$ \\
\hline Black (0/1) & $\begin{array}{c}0.439 \\
(0.496)\end{array}$ & $\begin{array}{c}0.302 \\
(0.459)\end{array}$ & $\begin{array}{c}0.438 \\
(0.496)\end{array}$ & $\begin{array}{c}0.303 \\
(0.460)\end{array}$ & $\begin{array}{c}0.442 \\
(0.497)\end{array}$ & $\begin{array}{c}0.287 \\
(0.453)\end{array}$ & $\begin{array}{c}0.492 \\
(0.500)\end{array}$ & $\begin{array}{c}0.290 \\
(0.454)\end{array}$ & $\begin{array}{c}0.359 \\
(0.480)\end{array}$ & $\begin{array}{c}0.267 \\
(0.442)\end{array}$ \\
\hline Other race $(0 / 1)$ & $\begin{array}{c}0.078 \\
(0.268)\end{array}$ & $\begin{array}{c}0.084 \\
(0.278)\end{array}$ & $\begin{array}{c}0.091 \\
(0.287)\end{array}$ & $\begin{array}{c}0.081 \\
(0.273)\end{array}$ & $\begin{array}{c}0.095 \\
(0.294)\end{array}$ & $\begin{array}{c}0.078 \\
(0.268)\end{array}$ & $\begin{array}{c}0.104 \\
(0.305)\end{array}$ & $\begin{array}{c}0.078 \\
(0.268)\end{array}$ & $\begin{array}{c}0.086 \\
(0.281)\end{array}$ & $\begin{array}{c}0.075 \\
(0.264)\end{array}$ \\
\hline Age in $2011^{1}$ & $\begin{array}{l}32.487 \\
(9.262)\end{array}$ & $\begin{array}{c}37.063 \\
(12.161)\end{array}$ & $\begin{array}{l}31.852 \\
(9.102)\end{array}$ & $\begin{array}{c}37.197 \\
(12.113)\end{array}$ & $\begin{array}{l}32.208 \\
(9.593)\end{array}$ & $\begin{array}{c}37.577 \\
(12.161)\end{array}$ & $\begin{array}{l}31.341 \\
(8.794)\end{array}$ & $\begin{array}{c}37.254 \\
(12.092)\end{array}$ & $\begin{array}{c}33.842 \\
(10.127)\end{array}$ & $\begin{array}{c}41.263 \\
(13.484)\end{array}$ \\
\hline Born in South & $\begin{array}{c}0.437 \\
(0.496)\end{array}$ & $\begin{array}{c}0.376 \\
(0.484)\end{array}$ & $\begin{array}{c}0.449 \\
(0.497)\end{array}$ & $\begin{array}{c}0.374 \\
(0.484)\end{array}$ & $\begin{array}{c}0.465 \\
(0.499)\end{array}$ & $\begin{array}{c}0.360 \\
(0.480)\end{array}$ & $\begin{array}{c}0.468 \\
(0.499)\end{array}$ & $\begin{array}{c}0.370 \\
(0.483)\end{array}$ & $\begin{array}{c}0.422 \\
(0.494)\end{array}$ & $\begin{array}{c}0.314 \\
(0.464)\end{array}$ \\
\hline \multicolumn{11}{|l|}{ Mother variables } \\
\hline High school completion & $\begin{array}{c}0.188 \\
(0.391)\end{array}$ & $\begin{array}{c}0.165 \\
(0.371)\end{array}$ & $\begin{array}{c}0.185 \\
(0.389)\end{array}$ & $\begin{array}{c}0.165 \\
(0.372)\end{array}$ & $\begin{array}{c}0.156 \\
(0.363)\end{array}$ & $\begin{array}{c}0.175 \\
(0.380)\end{array}$ & $\begin{array}{c}0.199 \\
(0.399)\end{array}$ & $\begin{array}{c}0.162 \\
(0.369)\end{array}$ & $\begin{array}{c}0.178 \\
(0.382)\end{array}$ & $\begin{array}{c}0.150 \\
(0.357)\end{array}$ \\
\hline Some college & $\begin{array}{c}0.138 \\
(0.345)\end{array}$ & $\begin{array}{c}0.094 \\
(0.292)\end{array}$ & $\begin{array}{c}0.154 \\
(0.361)\end{array}$ & $\begin{array}{c}0.090 \\
(0.287)\end{array}$ & $\begin{array}{c}0.129 \\
(0.335)\end{array}$ & $\begin{array}{c}0.094 \\
(0.292)\end{array}$ & $\begin{array}{c}0.142 \\
(0.349)\end{array}$ & $\begin{array}{c}0.094 \\
(0.292)\end{array}$ & $\begin{array}{c}0.120 \\
(0.325)\end{array}$ & $\begin{array}{c}0.065 \\
(0.246)\end{array}$ \\
\hline College completion & $\begin{array}{c}0.085 \\
(0.280)\end{array}$ & $\begin{array}{c}0.094 \\
(0.292)\end{array}$ & $\begin{array}{c}0.096 \\
(0.295)\end{array}$ & $\begin{array}{c}0.091 \\
(0.288)\end{array}$ & $\begin{array}{c}0.062 \\
(0.242)\end{array}$ & $\begin{array}{c}0.104 \\
(0.305)\end{array}$ & $\begin{array}{c}0.071 \\
(0.257)\end{array}$ & $\begin{array}{c}0.098 \\
(0.297)\end{array}$ & $\begin{array}{c}0.103 \\
(0.304)\end{array}$ & $\begin{array}{c}0.068 \\
(0.246)\end{array}$ \\
\hline
\end{tabular}




\begin{tabular}{|c|c|c|c|c|c|c|c|c|c|c|}
\hline \multicolumn{11}{|l|}{ Appendix A: Continued } \\
\hline Married @ child born & 0.864 & 0.934 & 0.858 & 0.935 & 0.838 & 0.951 & 0.674 & 0.984 & 0.894 & 0.983 \\
\hline & $(0.343)$ & $(0.248)$ & $(0.349)$ & $(0.247)$ & $(0.369)$ & $(0.215)$ & $(0.469)$ & $(0.125)$ & $(0.308)$ & (0.128) \\
\hline $\begin{array}{l}\text { Work in manufacturing } \\
@ \text { child born }\end{array}$ & $\begin{array}{c}0.053 \\
(0.225)\end{array}$ & $\begin{array}{c}0.040 \\
(0.197)\end{array}$ & $\begin{array}{c}0.050 \\
(0.218)\end{array}$ & $\begin{array}{c}0.041 \\
(0.199)\end{array}$ & $\begin{array}{c}0.037 \\
(0.190)\end{array}$ & $\begin{array}{c}0.045 \\
(0.208)\end{array}$ & $\begin{array}{c}0.038 \\
(0.191)\end{array}$ & $\begin{array}{c}0.044 \\
(0.206)\end{array}$ & $\begin{array}{c}0.039 \\
(0.195)\end{array}$ & $\begin{array}{c}0.051 \\
(0.221)\end{array}$ \\
\hline \multicolumn{11}{|l|}{ Father variables } \\
\hline High school completion & $\begin{array}{c}0.129 \\
(0.336)\end{array}$ & $\begin{array}{c}0.124 \\
(0.330)\end{array}$ & $\begin{array}{c}0.128 \\
(0.334)\end{array}$ & $\begin{array}{c}0.125 \\
(0.331)\end{array}$ & $\begin{array}{c}0.111 \\
(0.314)\end{array}$ & $\begin{array}{c}0.131 \\
(0.337)\end{array}$ & $\begin{array}{c}0.137 \\
(0.344)\end{array}$ & $\begin{array}{c}0.123 \\
(0.328)\end{array}$ & $\begin{array}{c}0.134 \\
(0.341)\end{array}$ & $\begin{array}{c}0.105 \\
(0.306)\end{array}$ \\
\hline \multirow[t]{2}{*}{ Some college } & 0.074 & 0.070 & 0.081 & 0.068 & 0.064 & 0.073 & 0.070 & 0.071 & 0.079 & 0.052 \\
\hline & $(0.263)$ & $(0.255)$ & $(0.273)$ & $(0.251)$ & $(0.246)$ & $(0.261)$ & $(0.254)$ & $(0.257)$ & $(0.270)$ & $(0.222)$ \\
\hline \multirow{2}{*}{ College completion } & 0.077 & 0.105 & 0.081 & 0.104 & 0.056 & 0.116 & 0.050 & 0.111 & 0.112 & 0.070 \\
\hline & $(0.267)$ & $(0.307)$ & $(0.273)$ & $(0.305)$ & $(0.231)$ & $(0.320)$ & $(0.218)$ & $(0.315)$ & $(0.315)$ & $(0.256)$ \\
\hline \multirow[t]{2}{*}{ Married @ child born } & 0.867 & 0.935 & 0.861 & 0.936 & 0.841 & 0.952 & 0.679 & 0.984 & 0.895 & 0.984 \\
\hline & $(0.339)$ & $(0.247)$ & $(0.346)$ & $(0.245)$ & $(0.365)$ & $(0.214)$ & $(0.467)$ & (0.124) & $(0.306)$ & (0.127) \\
\hline \multirow{3}{*}{$\begin{array}{l}\text { Work in manufacturing } \\
\text { @ child born }\end{array}$} & 0.127 & 0.165 & 0.119 & 0.167 & 0.126 & 0.169 & 0.079 & 0.176 & 0.129 & 0.222 \\
\hline & $(0.333)$ & $(0.371)$ & $(0.324)$ & $(0.373)$ & $(0.332)$ & $(0.375)$ & $(0.270)$ & $(0.381)$ & $(0.335)$ & $(0.416)$ \\
\hline & 2,420 & 8,962 & 2,357 & 9,025 & 3,153 & 8,229 & 2,241 & 9,141 & 7,935 & 3,447 \\
\hline
\end{tabular}




\begin{tabular}{|c|c|c|c|c|c|c|c|c|c|c|}
\hline \multicolumn{11}{|c|}{ Appendix B: Full Descriptive Statistics (events for fathers) } \\
\hline & \multicolumn{2}{|c|}{ Employment loss } & \multicolumn{2}{|c|}{ Employment gain } & \multicolumn{2}{|c|}{ Partner loss } & \multicolumn{2}{|c|}{ Partner gain } & \multicolumn{2}{|c|}{ Moved } \\
\hline & Yes & No & Yes & No & Yes & No & Yes & No & Yes & No \\
\hline & $\begin{array}{l}\text { Mean } \\
(S D)\end{array}$ & $\begin{array}{l}\text { Mean } \\
(S D)\end{array}$ & $\begin{array}{l}\text { Mean } \\
(S D)\end{array}$ & $\begin{array}{l}\text { Mean } \\
(S D)\end{array}$ & $\begin{array}{l}\text { Mean } \\
\text { (SD) }\end{array}$ & $\begin{array}{l}\text { Mean } \\
(S D)\end{array}$ & $\begin{array}{l}\text { Mean } \\
(S D)\end{array}$ & $\begin{array}{l}\text { Mean } \\
(S D)\end{array}$ & $\begin{array}{l}\text { Mean } \\
(S D)\end{array}$ & $\begin{array}{l}\text { Mean } \\
(S D)\end{array}$ \\
\hline \multicolumn{11}{|l|}{ Child variables } \\
\hline $\begin{array}{l}\text { High school completion } \\
\text { by age } 19\end{array}$ & $\begin{array}{c}0.470 \\
(0.499)\end{array}$ & $\begin{array}{c}0.488 \\
(0.500)\end{array}$ & $\begin{array}{c}0.470 \\
(0.499)\end{array}$ & $\begin{array}{c}0.488 \\
(0.500)\end{array}$ & $\begin{array}{c}0.460 \\
(0.498)\end{array}$ & $\begin{array}{c}0.493 \\
(0.500)\end{array}$ & $\begin{array}{c}0.453 \\
(0.498)\end{array}$ & $\begin{array}{c}0.491 \\
(0.500)\end{array}$ & $\begin{array}{c}0.505 \\
(0.500)\end{array}$ & $\begin{array}{c}0.436 \\
(0.496)\end{array}$ \\
\hline $\begin{array}{l}\text { College attendance } \\
\text { by age } 21\end{array}$ & $\begin{array}{c}0.225 \\
(0.417)\end{array}$ & $\begin{array}{c}0.269 \\
(0.444)\end{array}$ & $\begin{array}{c}0.221 \\
(0.415)\end{array}$ & $\begin{array}{c}0.269 \\
(0.444)\end{array}$ & $\begin{array}{c}0.214 \\
(0.411)\end{array}$ & $\begin{array}{l}0.276 \\
(0.447)\end{array}$ & $\begin{array}{c}0.206 \\
(0.405)\end{array}$ & $\begin{array}{c}0.272 \\
(0.445)\end{array}$ & $\begin{array}{c}0.271 \\
(0.444)\end{array}$ & $\begin{array}{l}0.232 \\
(0.422)\end{array}$ \\
\hline $\begin{array}{l}\text { College completion } \\
\text { by age } 25\end{array}$ & $\begin{array}{l}0.079 \\
(0.270)\end{array}$ & $\begin{array}{c}0.146 \\
(0.353)\end{array}$ & $\begin{array}{c}0.084 \\
(0.278)\end{array}$ & $\begin{array}{c}0.143 \\
(0.350)\end{array}$ & $\begin{array}{c}0.079 \\
(0.270)\end{array}$ & $\begin{array}{c}0.149 \\
(0.356)\end{array}$ & $\begin{array}{c}0.077 \\
(0.266)\end{array}$ & $\begin{array}{c}0.143 \\
(0.350)\end{array}$ & $\begin{array}{c}0.122 \\
(0.328)\end{array}$ & $\begin{array}{l}0.148 \\
(0.355)\end{array}$ \\
\hline Male (0/1) & $\begin{array}{c}0.521 \\
(0.500)\end{array}$ & $\begin{array}{c}0.509 \\
(0.500)\end{array}$ & $\begin{array}{c}0.514 \\
(0.500)\end{array}$ & $\begin{array}{c}0.511 \\
(0.500)\end{array}$ & $\begin{array}{c}0.510 \\
(0.500)\end{array}$ & $\begin{array}{c}0.513 \\
(0.500)\end{array}$ & $\begin{array}{c}0.521 \\
(0.500)\end{array}$ & $\begin{array}{l}0.510 \\
(0.500)\end{array}$ & $\begin{array}{c}0.515 \\
(0.500)\end{array}$ & $\begin{array}{c}0.505 \\
(0.500)\end{array}$ \\
\hline Black (0/1) & $\begin{array}{c}0.419 \\
(0.493)\end{array}$ & $\begin{array}{c}0.304 \\
(0.460)\end{array}$ & $\begin{array}{c}0.427 \\
(0.495)\end{array}$ & $\begin{array}{c}0.304 \\
(0.460)\end{array}$ & $\begin{array}{c}0.443 \\
(0.497)\end{array}$ & $\begin{array}{c}0.287 \\
(0.453)\end{array}$ & $\begin{array}{c}0.484 \\
(0.500)\end{array}$ & $\begin{array}{c}0.293 \\
(0.455)\end{array}$ & $\begin{array}{c}0.357 \\
(0.479)\end{array}$ & $\begin{array}{c}0.270 \\
(0.444)\end{array}$ \\
\hline Other race $(0 / 1)$ & $\begin{array}{c}0.099 \\
(0.299)\end{array}$ & $\begin{array}{c}0.078 \\
(0.268)\end{array}$ & $\begin{array}{c}0.103 \\
(0.304)\end{array}$ & $\begin{array}{c}0.077 \\
(0.267)\end{array}$ & $\begin{array}{c}0.095 \\
(0.293)\end{array}$ & $\begin{array}{c}0.078 \\
(0.269)\end{array}$ & $\begin{array}{c}0.090 \\
(0.287)\end{array}$ & $\begin{array}{c}0.081 \\
(0.273)\end{array}$ & $\begin{array}{c}0.086 \\
(0.281)\end{array}$ & $\begin{array}{c}0.075 \\
(0.263)\end{array}$ \\
\hline Age in 2011 & $\begin{array}{l}32.882 \\
(8.773)\end{array}$ & $\begin{array}{c}37.061 \\
(12.356)\end{array}$ & $\begin{array}{l}32.656 \\
(8.643)\end{array}$ & $\begin{array}{c}37.055 \\
(12.320)\end{array}$ & $\begin{array}{l}32.152 \\
(9.560)\end{array}$ & $\begin{array}{c}37.588 \\
(12.159)\end{array}$ & $\begin{array}{l}31.640 \\
(8.822)\end{array}$ & $\begin{array}{c}37.164 \\
(12.117)\end{array}$ & $\begin{array}{c}33.913 \\
(10.141)\end{array}$ & $\begin{array}{c}41.055 \\
(13.540)\end{array}$ \\
\hline Born in South & $\begin{array}{c}0.403 \\
(0.490)\end{array}$ & $\begin{array}{c}0.385 \\
(0.487)\end{array}$ & $\begin{array}{c}0.404 \\
(0.491)\end{array}$ & $\begin{array}{c}0.385 \\
(0.487)\end{array}$ & $\begin{array}{c}0.461 \\
(0.499)\end{array}$ & $\begin{array}{c}0.362 \\
(0.481)\end{array}$ & $\begin{array}{c}0.461 \\
(0.499)\end{array}$ & $\begin{array}{c}0.372 \\
(0.483)\end{array}$ & $\begin{array}{c}0.420 \\
(0.494)\end{array}$ & $\begin{array}{c}0.319 \\
(0.466)\end{array}$ \\
\hline \multicolumn{11}{|l|}{ Mother variables } \\
\hline High school completion & $\begin{array}{c}0.190 \\
(0.392)\end{array}$ & $\begin{array}{c}0.163 \\
(0.370)\end{array}$ & $\begin{array}{c}0.193 \\
(0.394)\end{array}$ & $\begin{array}{c}0.163 \\
(0.369)\end{array}$ & $\begin{array}{c}0.156 \\
(0.363)\end{array}$ & $\begin{array}{c}0.175 \\
(0.380)\end{array}$ & $\begin{array}{c}0.170 \\
(0.375)\end{array}$ & $\begin{array}{c}0.169 \\
(0.375)\end{array}$ & $\begin{array}{c}0.176 \\
(0.381)\end{array}$ & $\begin{array}{c}0.154 \\
(0.361)\end{array}$ \\
\hline Some college & $\begin{array}{c}0.128 \\
(0.335)\end{array}$ & $\begin{array}{c}0.096 \\
(0.295)\end{array}$ & $\begin{array}{c}0.129 \\
(0.336)\end{array}$ & $\begin{array}{c}0.096 \\
(0.295)\end{array}$ & $\begin{array}{c}0.127 \\
(0.334)\end{array}$ & $\begin{array}{c}0.094 \\
(0.292)\end{array}$ & $\begin{array}{c}0.122 \\
(0.328)\end{array}$ & $\begin{array}{c}0.099 \\
(0.299)\end{array}$ & $\begin{array}{c}0.120 \\
(0.324)\end{array}$ & $\begin{array}{c}0.067 \\
(0.250)\end{array}$ \\
\hline College completion & $\begin{array}{c}0.053 \\
(0.225)\end{array}$ & $\begin{array}{c}0.104 \\
(0.305)\end{array}$ & $\begin{array}{c}0.052 \\
(0.222)\end{array}$ & $\begin{array}{c}0.104 \\
(0.305)\end{array}$ & $\begin{array}{c}0.063 \\
(0.242)\end{array}$ & $\begin{array}{c}0.104 \\
(0.305)\end{array}$ & $\begin{array}{c}0.059 \\
(0.236)\end{array}$ & $\begin{array}{c}0.100 \\
(0.300)\end{array}$ & $\begin{array}{c}0.104 \\
(0.305)\end{array}$ & $\begin{array}{c}0.067 \\
(0.250)\end{array}$ \\
\hline Married@ child born & $\begin{array}{c}0.866 \\
(0.341)\end{array}$ & $\begin{array}{c}0.935 \\
(0.247)\end{array}$ & $\begin{array}{c}0.860 \\
(0.346)\end{array}$ & $\begin{array}{c}0.935 \\
(0.246)\end{array}$ & $\begin{array}{c}0.838 \\
(0.368)\end{array}$ & $\begin{array}{c}0.951 \\
(0.215)\end{array}$ & $\begin{array}{c}0.673 \\
(0.469)\end{array}$ & $\begin{array}{c}0.983 \\
(0.128)\end{array}$ & $\begin{array}{c}0.895 \\
(0.306)\end{array}$ & $\begin{array}{c}0.978 \\
(0.147)\end{array}$ \\
\hline $\begin{array}{c}\text { Work in manufacturing } \\
@ \text { child born }\end{array}$ & $\begin{array}{l}0.041 \\
(0.198)\end{array}$ & $\begin{array}{l}0.044 \\
(0.205)\end{array}$ & $\begin{array}{l}0.042 \\
(0.202)\end{array}$ & $\begin{array}{c}0.043 \\
(0.204)\end{array}$ & $\begin{array}{r}0.037 \\
(0.187)\end{array}$ & $\begin{array}{r}0.045 \\
(0.208)\end{array}$ & $\begin{array}{l}0.027 \\
(0.162)\end{array}$ & $\begin{array}{l}0.047 \\
(0.212)\end{array}$ & $\begin{array}{l}0.039 \\
(0.195)\end{array}$ & $\begin{array}{l}0.052 \\
(0.221)\end{array}$ \\
\hline
\end{tabular}




\begin{tabular}{|c|c|c|c|c|c|c|c|c|c|c|c|}
\hline \multicolumn{12}{|l|}{ Appendix B: Continued } \\
\hline \multicolumn{12}{|l|}{ Father variables } \\
\hline \multirow[t]{2}{*}{ High school completion } & & 0.166 & 0.113 & 0.177 & 0.111 & 0.112 & 0.131 & 0.154 & 0.118 & 0.136 & 0.102 \\
\hline & & $(0.372)$ & $(0.317)$ & $(0.382)$ & $(0.314)$ & $(0.315)$ & $(0.337)$ & $(0.361)$ & $(0.323)$ & $(0.342)$ & $(0.303)$ \\
\hline \multirow[t]{2}{*}{ Some college } & & 0.064 & 0.073 & 0.065 & 0.072 & 0.067 & 0.072 & 0.096 & 0.065 & 0.079 & 0.052 \\
\hline & & $(0.245)$ & $(0.260)$ & $(0.247)$ & $(0.259)$ & $(0.250)$ & $(0.259)$ & $(0.295)$ & $(0.246)$ & $(0.270)$ & $(0.222)$ \\
\hline \multirow[t]{2}{*}{ College completion } & & 0.052 & 0.114 & 0.056 & 0.111 & 0.058 & 0.115 & 0.065 & 0.107 & 0.112 & 0.070 \\
\hline & & $(0.222)$ & $(0.317)$ & $(0.230)$ & $(0.315)$ & $(0.233)$ & $(0.319)$ & $(0.247)$ & $(0.310)$ & $(0.315)$ & $(0.256)$ \\
\hline \multirow[t]{2}{*}{ Married@ child born } & & 0.867 & 0.936 & 0.860 & 0.937 & 0.841 & 0.952 & 0.674 & 0.985 & 0.897 & 0.980 \\
\hline & & $(0.340)$ & $(0.244)$ & $(0.347)$ & $(0.242)$ & $(0.365)$ & $(0.214)$ & $(0.469)$ & $(0.121)$ & $(0.304)$ & (0.141) \\
\hline \multirow{3}{*}{$\begin{array}{l}\text { Work in manufacturing } \\
\text { @ child born }\end{array}$} & & 0.149 & 0.160 & 0.143 & 0.161 & 0.125 & 0.169 & 0.095 & 0.171 & 0.131 & 0.216 \\
\hline & & $(0.356)$ & $(0.366)$ & $(0.350)$ & $(0.367)$ & $(0.331)$ & $(0.375)$ & $(0.294)$ & $(0.377)$ & $(0.337)$ & $(0.412)$ \\
\hline & $N$ & 2,645 & 8,737 & 2,497 & 8,885 & 3,137 & 8,245 & 2,213 & 9,169 & 7,913 & 3,469 \\
\hline
\end{tabular}




\begin{tabular}{|c|c|c|c|c|c|c|c|c|c|c|c|c|}
\hline \multicolumn{13}{|c|}{ Appendix C: Effects of single and combined events on children's educational outcomes, events for mothers } \\
\hline & \multicolumn{4}{|c|}{$\begin{array}{c}\text { Completed high school } \\
\text { by age } 19\end{array}$} & \multicolumn{4}{|c|}{$\begin{array}{c}\text { Attended college } \\
\text { by age } 21\end{array}$} & \multicolumn{4}{|c|}{$\begin{array}{c}\text { College completion } \\
\text { by age } 25\end{array}$} \\
\hline & \multicolumn{2}{|c|}{$\begin{array}{l}\text { With child } \\
\text { covariates }\end{array}$} & \multicolumn{2}{|c|}{$\begin{array}{l}\text { With child, } \\
\text { mother \& } \\
\text { father } \\
\text { covariates }\end{array}$} & \multicolumn{2}{|c|}{$\begin{array}{l}\text { With child } \\
\text { covariates }\end{array}$} & \multicolumn{2}{|c|}{$\begin{array}{c}\text { With child, } \\
\text { mother \& } \\
\text { father } \\
\text { covariates }\end{array}$} & \multicolumn{2}{|c|}{$\begin{array}{l}\text { With child } \\
\text { covariates }\end{array}$} & \multicolumn{2}{|c|}{$\begin{array}{c}\text { With child, } \\
\text { mother \& } \\
\text { father } \\
\text { covariates }\end{array}$} \\
\hline \multicolumn{13}{|l|}{ Single events } \\
\hline Lost employment & 0.087 & $\dagger$ & 0.057 & & -0.075 & & -0.073 & & -0.133 & $\dagger$ & -0.067 & \\
\hline Gained employment & 0.101 & * & 0.033 & & -0.120 & $*$ & -0.181 & $* *$ & -0.095 & & -0.082 & \\
\hline Lost partner & -0.106 & $*$ & 0.030 & & -0.388 & $* * *$ & -0.188 & $* *$ & -0.556 & $* * *$ & -0.243 & $* *$ \\
\hline Gained partner & -0.088 & + & -0.048 & & -0.376 & $* * *$ & -0.287 & $* * *$ & -0.580 & $* * *$ & -0.336 & $* *$ \\
\hline Moved & 0.432 & $* * *$ & 0.418 & $* * *$ & 0.161 & $* *$ & 0.066 & & -0.078 & & -0.269 & $* * *$ \\
\hline \multicolumn{13}{|l|}{ Event combinations } \\
\hline Lost employment + Moved & -0.192 & $* *$ & -0.228 & $* *$ & -0.316 & $* * *$ & -0.305 & $* * *$ & -0.466 & $* * *$ & -0.370 & $* *$ \\
\hline Gained employment + Moved & -0.068 & & -0.130 & + & -0.296 & $* * *$ & -0.335 & $* * *$ & -0.427 & $* * *$ & -0.415 & $* *$ \\
\hline Lost partner + Moved & -0.238 & $* * *$ & -0.155 & $* *$ & -0.503 & $* * *$ & -0.365 & $* * *$ & -0.663 & $* * *$ & -0.378 & $* * *$ \\
\hline Gained partner + Moved & -0.127 & $*$ & -0.086 & & -0.402 & $* * *$ & -0.312 & $* * *$ & -0.566 & $* * *$ & -0.344 & $* *$ \\
\hline Gained employment + Gained partner & -0.233 & $\dagger$ & -0.150 & & -0.578 & $* *$ & -0.406 & $*$ & -1.027 & $* *$ & -0.650 & $\dagger$ \\
\hline Gained employment + Lost partner & -0.309 & $*$ & -0.348 & $*$ & -0.650 & $* * *$ & -0.556 & $* *$ & -0.324 & & -0.003 & \\
\hline Lost employment + Lost partner & -0.492 & $* * *$ & -0.407 & $* *$ & -0.904 & $* * *$ & -0.725 & $* * *$ & -0.639 & $*$ & -0.256 & \\
\hline Lost employment + Gained partner & -0.361 & $*$ & -0.261 & & -0.283 & & -0.090 & & -0.912 & $* *$ & -0.604 & $\dagger$ \\
\hline \multicolumn{13}{|c|}{$\begin{array}{l}\text { Notes: Each coefficient represents a separate model; coefficients are reported as log-odds. Only events that occurred when the child was } \\
\text { between } 1 \text { and } 17 \text { years old were included. Children must be at least } 19 \text { years old by } 2011 \text { to be included in the sample }(\mathrm{N}=11,382) \text {. For } \\
\text { event combinations, the order of events was unknown, and the two events occurred within the same two-year period. All models included } \\
\text { controls for child's sex, child's race, child's age in 2011, child born in the South, mother's and father's education, mother's and father's } \\
\text { marital status at child's birth, and mother's and father's job in manufacturing. } \\
+p<.10^{*} p<.05^{* *} p<.011^{* * *} p<.001 \text { (two-tailed tests) }\end{array}$} \\
\hline
\end{tabular}




\begin{tabular}{|c|c|c|c|c|c|c|c|c|c|c|c|c|}
\hline \multicolumn{13}{|c|}{ Appendix D: Effects of single and combined events on children's educational outcomes, events for fathers } \\
\hline & \multicolumn{4}{|c|}{$\begin{array}{l}\text { Completed high school } \\
\text { by age } 19\end{array}$} & \multicolumn{4}{|c|}{$\begin{array}{c}\text { Attended college } \\
\text { by age } 21\end{array}$} & \multicolumn{4}{|c|}{$\begin{array}{c}\text { College completion } \\
\text { by age } 25\end{array}$} \\
\hline & \multicolumn{2}{|c|}{$\begin{array}{l}\text { With child } \\
\text { covariates }\end{array}$} & \multicolumn{2}{|c|}{$\begin{array}{l}\text { With child, } \\
\text { mother \& } \\
\text { father } \\
\text { covariates }\end{array}$} & \multicolumn{4}{|c|}{$\begin{array}{l}\text { With child, } \\
\text { mother \& } \\
\text { father } \\
\text { covariates }\end{array}$} & \multicolumn{2}{|c|}{$\begin{array}{l}\text { With child } \\
\text { covariates }\end{array}$} & \multicolumn{2}{|c|}{$\begin{array}{c}\text { With child, } \\
\text { mother \& } \\
\text { father } \\
\text { covariates }\end{array}$} \\
\hline \multicolumn{13}{|l|}{ Single events } \\
\hline Lost employment & -0.025 & & 0.021 & & -0.263 & $* * *$ & -0.142 & $*$ & -0.550 & $* * *$ & -0.288 & $* *$ \\
\hline Gained employment & -0.032 & & -0.010 & & -0.296 & $* * *$ & -0.189 & $* *$ & -0.448 & $* * *$ & -0.190 & $*$ \\
\hline Lost partner & -0.100 & $*$ & 0.042 & & -0.388 & $* * *$ & -0.188 & $* *$ & -0.552 & $* * *$ & -0.248 & $* *$ \\
\hline Gained partner & -0.119 & $*$ & -0.043 & & -0.405 & $* * *$ & -0.280 & $* * *$ & -0.501 & $* * *$ & -0.273 & $* *$ \\
\hline Moved & 0.400 & $* * *$ & 0.383 & $* * *$ & 0.186 & $* * *$ & 0.107 & $\dagger$ & -0.032 & & -0.218 & $* *$ \\
\hline \multicolumn{13}{|l|}{ Event combinations } \\
\hline Lost employment + Moved & -0.278 & $* * *$ & -0.236 & $* *$ & -0.406 & $* * *$ & -0.260 & $* *$ & -0.753 & $* * *$ & -0.511 & $* * *$ \\
\hline Gained employment + Moved & -0.284 & $* * *$ & -0.224 & $* *$ & -0.421 & $* * *$ & -0.289 & $* *$ & -0.657 & $* * *$ & -0.408 & $* *$ \\
\hline Lost partner + Moved & -0.175 & $* * *$ & -0.094 & + & -0.460 & $* * *$ & -0.317 & $* * *$ & -0.620 & $* * *$ & -0.375 & $* * *$ \\
\hline Gained partner + Moved & -0.175 & $* *$ & -0.077 & & -0.437 & $* * *$ & -0.292 & $* * *$ & -0.486 & $* * *$ & -0.260 & $*$ \\
\hline Gained employment + Gained partner & -0.350 & $* *$ & -0.159 & & -0.314 & $*$ & 0.017 & & -0.562 & $*$ & -0.023 & \\
\hline Gained employment + Lost partner & -0.187 & & -0.074 & & -0.799 & $* * *$ & -0.571 & $* *$ & -0.447 & $\dagger$ & -0.050 & \\
\hline Lost employment + Lost partner & -0.472 & $* * *$ & -0.364 & $* *$ & -0.680 & $* * *$ & -0.477 & $* *$ & -0.685 & $* *$ & -0.378 & \\
\hline Lost employment + Gained partner & -0.355 & $* *$ & -0.249 & + & -0.582 & $* *$ & -0.270 & & -0.616 & $*$ & -0.096 & \\
\hline \multicolumn{13}{|c|}{$\begin{array}{l}\text { Notes: Each coefficient represents a separate model; coefficients are reported as log-odds. Only events that occurred when the child was } \\
\text { between } 1 \text { and } 17 \text { years old were included. Children must be at least } 19 \text { years old by } 2011 \text { to be included in the sample }(\mathrm{N}=11,382) \text {. For } \\
\text { event combinations, the order of events was unknown, and the two events occurred within the same two-year period. All models included } \\
\text { controls for child's sex, child's race, child's age in } 2011 \text {, child born in the South, mother's and father's education, mother's and father's } \\
\text { marital status at child's birth, and mother's and father's job in manufacturing. } \\
+p<.10^{*} p<.05{ }^{* *} p<.011^{* * *} p<.001 \text { (two-tailed tests) }\end{array}$} \\
\hline
\end{tabular}

